

Б. В. Карпов, Д. Ю. Ногин, Трехблочные исключительные наборы на поверхностях дель Пеццо, Изв. РАН. Сер. матем., 1998, том 62, выпуск 3, 3-38

DOI: https://doi.org/10.4213/im205

Использование Общероссийского математического портала Math-Net.Ru подразумевает, что вы прочитали и согласны с пользовательским соглашением

http://www . mathnet.ru/rus/agreement

Параметры загрузки:

IP : 54.198 .55 .26

26 апреля 2023 г., $16: 19: 28$ 
УДК 512.723

\author{
Б. В. Карпов, Д. Ю. Ногин
}

\title{
Трехблочные исключительные наборы на поверхностях дель Пеццо
}

\begin{abstract}
В работе изучаются полные исключительные наборы когерентных пучков на поверхностях дель Пеццо, состоящие из трех блоков, внутри каждого из которых все взаимные Ext-группы между пучками нулевые. Мы показываем, что ранги пучков, входящих в один блок, одинаковы и что три ранга, отвечающие полному трехблочному исключительному набору, удовлетворяют диофантову уравнению типа Маркова, квадратичному по каждой переменной. Этих уравнений конечное число для каждой поверхности дель Пеццо, их полный список приводится. Мы также описываем действие на множестве полных трехблочных исключительных наборов группы кос из трех нитей посредством перестроек. В частности, в любой орбите есть трехблочный набор с суммой рангов, минимальной для решений данного уравнения типа Маркова, и все орбиты получаются друг из друга подкруткой на обратимый пучок и действием группы Вейля. Это позволяет вычислить число орбит с точностью до подкрутки.

Библиография: 19 наименований.
\end{abstract}

\section{Введение}

Напомним, что пучок $E$ называется исключительным, если $\operatorname{Hom}(E, E) \cong \mathbb{C}$ и $\operatorname{Ext}^{i}(E, E)=0$ при $i>0$. Упорядоченный набор пучков $\left(E_{1}, \ldots, E_{\alpha}\right)$ называется исключительным, если все $E_{j}$ исключительны и $\operatorname{Ext}^{i}\left(E_{k}, E_{j}\right)=0 \forall i, \forall k>j$.

Теория исключительных пучков и исключительных наборов развивается уже в течение десяти лет. Впервые исключительные расслоения на $\mathbb{P}^{2}$ появились в работе [6] в 1985 г. Там с помощью дискретных инвариантов $\left(r, c_{1}, c_{2}\right)$ исключительных расслоений построена граница множества этих инвариантов для полустабильных пучков на $\mathbb{P}^{2}$ (здесь $r$ - ранг, $c_{1}, c_{2}$ - классы Чженя). В последующих работах [7]-[9] исключительные расслоения использовались для изучения многообразий модулей полустабильных пучков на $\mathbb{P}^{2}$.

В другом направлении теория исключительных пучков развивалась в Москве участниками семинара под руководством А.Н. Рудакова и А.Н. Тюрина. Первоначально общая постановка задачи была следующая: описать разумным образом множество исключительных пучков на данном многообразии. Для этой цели использовались перестройки исключительных пучков, дающие новые исключительные пучки, и было введено понятие спирали, позволяющее не заботиться о существовании перестроек [3]. Первые и наиболее яркие результаты были получены на $\mathbb{P}^{2}[4]$ : любой исключительный пучок содержится в некоторой спирали,

Работа написана при частичной поддержке Международного научного фонда (гранты ISF № MKU 300 и ISF № MPN 000) и Российского фонда фундаментальных исследований (проекты 95-01-00840, 93-012-458, 96-01-01378 и 96-01-01323). 
а любая спираль получается конечным числом перестроек из спирали, состоящей из обратимых пучков. Возможность получения данного исключительного набора перестройками из некоторого простейшего набора канонического вида (на $\mathbb{P}^{2}-$ из набора, состоящего из обратимых пучков) называется конструктивностью. В основе доказательства конструктивности спиралей на $\mathbb{P}^{2}$ лежит следуюший факт: ранги $x, y, z$ трех подряд идущих расслоений в спирали удовлетворяют уравнению Маркова

$$
x^{2}+y^{2}+z^{2}=3 x y z
$$

причем перестройки спирали в точности соответствуют перестройкам решений этого уравнения. Сами же решения в натуральных числах, как показал А. А. Марков в [15], приводятся перестройками к решению $(1,1,1)$ с минимальной суммой $x+y+z$.

Такое соответствие является в каком-то смысле уникальным: во всех известных, кроме $\mathbb{P}^{2}$, случаях нет единого диофантова уравнения на ранги членов витка спирали. Поэтому для решения проблемы конструктивности на других многообразиях использовались иные методы. Так, данное А. Н. Рудаковым в [19] доказательство конструктивности спиралей и исключительных расслоений на $\mathbb{P}^{1} \times \mathbb{P}^{1}$ использовало настолько изяшные геометрические построения на плоскости $\operatorname{Pic}\left(\mathbb{P}^{1} \times \mathbb{P}^{1}\right) \otimes \mathbb{Q}$, что применить их в случае других многообразий не удается. В этой же работе были исследованы симметричные спирали, т.е. инвариантные относительно инволюции, переставляющей системы образующих $\mathbb{P}^{1} \times \mathbb{P}^{1}$. Из четырех расслоений, составляюших симметричный виток, два являются инвариантньми относительно инволюции, а два других при инволюции переходят друг в друга. Если $x$ и $y-$ ранги первых двух, а z-ранг последних двух расслоений, то, как показано в [19], тройка чисел $(x, y, z)$ является решением диофантова уравнения

$$
x^{2}+y^{2}+2 z^{2}=4 x y z,
$$

все решения которого в натуральных числах также приводятся перестройками к решению $(1,1,1)$.

Теми же методами С. Ю. Зюзина [10] доказала конструктивность исключительных пар на $\mathbb{P}^{1} \times \mathbb{P}^{1}$, т.е. утверждение о том, что любая исключительная пара содержится в некоторой спирали и тем самым может быть получена перестройками обратимых пучков.

Одним из авторов была разработана техника, позволяющая доказывать конструктивность не самих исключительных наборов, а их образов в группе Гротендика $\mathrm{K}_{0}$. Особенно хорошо она работала для многообразий, имеющих $\mathrm{rk}_{0}=4$ : рациональных линейчатых поверхностей, $\mathbb{P}^{3}$, трехмерной квадрики и многообразий Фано $V_{5}$ и $V_{22}$ (см. [16], [17]).

На сегодняшний день более эффективным оказался подход С. А. Кулешова, использующий жесткие $\left(\operatorname{Ext}^{1}(F, F)=0\right)$ и сверхжесткие $\left(\operatorname{Ext}^{i}(F, F)=0, i>0\right)$ пучки. Так, на поверхностях дель Пецц любой жесткий пучок изоморфен прямой сумме исключительных. На основе этого факта в [11] доказана конструктивность исключительных наборов любой длины на поверхностях дель Пещю. Обобщению этого результата для поверхностей с антиканоническим классом без базисных компонент посвящена работа [12]. С помощью такого подхода С. А. Кулешову удалось получить новое решение проблемы конструктивности на $\mathbb{P}^{2}$, не используюшее 
уравнение Маркова (см. [13]), что может открыть перспективы для исследования случая $\mathbb{P}^{n}$.

Целью настоящей статьи является описание класса таких исключительных наборов на поверхностях дель Пецц, для которых существуют уравнения типа Маркова, играюшие ту же роль, что и уравнения (1) и (2) на $\mathbb{P}^{2}$ и $\mathbb{P}^{1} \times \mathbb{P}^{1}$ соответственно. Это трехблочные исключительные наборы.

Мы будем называть блоком исключительный набор $\mathscr{E}=\left(E_{1}, \ldots, E_{\alpha}\right)$ такой, что $\operatorname{Ext}^{i}\left(E_{j}, E_{k}\right)=0 \forall i, \forall j \neq k$. В $\S 1$ мы показываем, что ранги пучков, входящих в блок, равны; это число будет обозначаться $r(\mathscr{E})$. Трехблочный набор - это исключительный набор

$$
(\mathscr{E}, \mathscr{F}, \mathscr{G})=\left(E_{1}, \ldots, E_{\alpha}, F_{1}, \ldots, F_{\beta}, G_{1}, \ldots, G_{\gamma}\right)
$$

состоящий из трех блоков. Перестройки таких наборов, сохраняющие трехблочную структуру, мы описываем в $\S 2$. Эти перестройки задают действие группы кос из трех нитей на множестве трехблочных наборов (см. п. 2.6).

Объектом нашего дальнейшего изучения являются не все трехблочные наборы, а только полные, т.е. порождаюшие производную категорию когерентных пучков на данной поверхности. Основной результат $\S 3$ состоит в том, что ранги $x=r(\mathscr{E}), y=r(\mathscr{F}), z=r(\mathscr{G})$ полного трехблочного набора $(\mathscr{E}, \mathscr{F}, \mathscr{G})$ удовлетворяют уравнению типа Маркова

$$
\alpha x^{2}+\beta y^{2}+\gamma z^{2}=\sqrt{K^{2} \alpha \beta \gamma} x y z
$$

где $K^{2}$ обозначает квадрат канонического класса поверхности. Коэффициентами левой части, как легко видеть, являются количества пучков в блоках $\mathscr{E}, \mathscr{F}, \mathscr{G}$. В правой части коэффициент $\sqrt{K^{2} \alpha \beta \gamma}$ - целое число. Это накладывает существенные ограничения на $\alpha, \beta$ и $\gamma$, благодаря чему полный список возможных уравнений (3) на поверхностях дель Пеццо получается вполне обозримым. Мы приводим его в п. 3.5. Там же мы показываем, что любое решение в натуральных числах каждого из уравнений (3) получается перестройками из минимального решения, т.е. имеющего минимальную сумму $x+y+z$. Это означает, что любой трехблочный набор с данными $\alpha, \beta$ и $\gamma$ может быть получен перестройками, сохраняющими трехблочную структуру, из набора с теми же $\alpha, \beta$ и $\gamma$ и минимальной суммой рангов. Отметим, что уравнения (1) и (2) являются частными случаями (3).

В $\S 4$ мы показываем, что для каждого уравнения (3) сушествует полный трехблочный набор с данными $\alpha, \beta$ и $\gamma$, отвечаюший минимальному решению этого уравнения. Кроме того, любые два таких набора получаются друг из друга действием группы Вейля и тензорным умножением (подкруткой) на обратимый пучок (§5). Отсюда следует, что если объединить в один класс орбиты действия группы кос на множестве полных трехблочных наборов, отличаюшиеся друг от друга подкруткой, то число классов, отвечающих фиксированному уравнению (3), окажется конечным. Эти числа подсчитаны в п. 5.7.

Авторы признательны А. Л. Городенцеву и С. А. Кулешову за внимание и интерес к данной теме. 


\section{§1. Предварительные сведения}

1.1. Обозначения и соглашения. Мы всегда будем считать $S$ поверхностью дель Пеццо над $\mathbb{C}$. По определению (см. $[14$, гл. IV]) $S$ - гладкая бирационально тривиальная поверхность с обильным антиканоническим классом $\left(-K_{S}\right)$.

Для когерентного пучка $F$ будут использоваться следуюшие дискретные инварианты: $r(F), c_{1}(F), c_{2}(F)$ - ранг и классы Чженя, $\mu(F)=\frac{c_{1}(F) \cdot\left(-K_{S}\right)}{r(F)}-$ наклон, $\nu(F)=\frac{c_{1}(F)}{r(F)}-$ точка в $\operatorname{Pic}(S) \otimes \mathbb{Q}, d(F)=c_{1}(F) \cdot\left(-K_{S}\right)$.

Для любых двух когерентных пучков $E$ и $F$ обозначим альтернированную сумму через

$$
\chi(E, F)=\sum_{i}(-1)^{i} \operatorname{dim} \operatorname{Ext}^{i}(E, F) .
$$

Она задает билинейную форму на $\mathrm{K}_{0}(S)$. Для пучков без кручения имеет место следуюшая формула, вытекаюшая из теоремы Римана-Роха:

$$
\chi(E, F)=r(E) r(F)\left(\chi\left(\mathscr{O}_{S}\right)+\frac{\mu(F)-\mu(E)}{2}+q(F)+q(E)-\frac{c_{1}(E) \cdot c_{1}(F)}{r(E) r(F)}\right) .
$$

Здесь $q(F)=\frac{c_{1}^{2}(F)-2 c_{2}(F)}{2 r(F)}=\frac{\operatorname{ch}_{2}(F)}{r(F)}$. Отметим, что $\chi\left(\mathscr{O}_{S}\right)=1$ для поверхностей дель Пещо.

Из существования локально свободной резольвенты для любого когерентного пучка и аддитивности обеих частей формулы (4) вытекает справедливость этой формулы для любых двух когерентных пучков. Раскрывая скобки, имеем

$$
\begin{aligned}
\chi(E, F)= & r(E) r(F) \chi\left(\mathscr{O}_{S}\right)+\frac{r(E) d(F)}{2}-\frac{r(F) d(E)}{2} \\
& +r(E) \operatorname{ch}_{2}(F)+r(F) \operatorname{ch}_{2}(E)-c_{1}(E) \cdot c_{1}(F) .
\end{aligned}
$$

Непосредственно из (4) следует выражение для кососимметрической части формы $\chi$ :

$$
\chi_{-}(E, F) \triangleq \chi(E, F)-\chi(F, E)=r(E) r(F)(\mu(F)-\mu(E)) .
$$

Если хотя бы один из рангов $r(E)$ или $r(F)$ нулевой, то можно пользоваться другой записью этой формулы:

$$
\chi_{-}(E, F)=\left|\begin{array}{ll}
r(E) & r(F) \\
d(E) & d(F)
\end{array}\right|
$$

Ограниченная производная категория когерентных пучков на $S$ будет обозначаться $D^{b}(S)$. Адлитивные функции, в частности $r$ и $d$, допускают естественное продолжение на $D^{b}(S)$. Именно, если $A$ - объект производной категории, представленный комплексом $K^{\cdot}$ с когомологическими пучками $H^{i}\left(K^{*}\right)$, и $s$ - аддитивная функция, то

$$
s(A)=\sum_{i}(-1)^{i} s\left(K^{i}\right)=\sum_{i}(-1)^{i} s\left(H^{i}\left(K^{\cdot}\right)\right) .
$$


1.2. Лемма. Пусть $0 \rightarrow E \rightarrow F \rightarrow G \rightarrow 0$ - точная тройка пучков на $S$; mогда $\chi_{-}(E, F)=\chi_{-}(F, G)=\chi_{-}(E, G)$.

Утверждение леммы немедленно следует из формулы (7).

1.3. Исключительные пучки. Напомним, что пучок $E$ называется исключительнымм, если $\operatorname{Hom}(E, E) \cong \mathbb{C}, \operatorname{Ext}^{i}(E, E)=0, i>0$.

В [11] доказано, что исключительный пучок на поверхности дель Пеццо либо локально свободен, либо является пучком кручения вида $\mathscr{O}_{\ell}(m)$, где $m \in \mathbb{Z}$ и $\ell$ исключительная кривая (или $(-1)$-кривая), т.е. неприводимая рациональная кривая, имеюшая $\ell^{2}=\ell \cdot K_{S}=-1$.

Исключительное расслоение стабильно по Мамфорду-Такемото относительно $\left(-K_{S}\right)$, и по этой причине однозначно с точностью до изоморфизма определяется своей точкой $\nu$ (см. [5]). С другой стороны, вычисления показывают, что $\mathrm{ch}_{2}\left(\mathscr{O}_{\ell}(m)\right)=\frac{1}{2}-m$. Таким образом, справедливо следуюшее утверждение.

ПРЕДЛОЖЕНИЕ. Исключительный пучок на поверхности дель Пецио $S$ однозначно с точностью до изоморфизма определяется своим образом в $\mathrm{K}_{0}(S)$.

Понятие исключительности естественньм образом обобщается на производную категорию. Объект $A \in D^{b}(S)$ называется исключительныцм, если $\operatorname{Hom}_{D^{b}(S)}^{0}(A, A) \cong \mathbb{C}, \operatorname{Hom}_{D^{b}(S)}^{i}(A, A)=0, \quad i \neq 0$. Известно (см. [11]), что на поверхности дель Пещо объект производной категории является исключительным тогда и только тогда, когда он изоморфен $\delta E[i]-$ сдвинутому в $D^{b}(S)$ образу исключительного пучка $E$ при каноническом вложении $\delta$ основной категории в производную.

Определим наклон исключительного пучка кручения, положив

$$
\mu\left(\mathscr{O}_{\ell}(m)\right)=+\infty
$$

1.4. Лемма. Пусть $E, F$ - исключительнье пучки на $S$; тогда

$$
\operatorname{sgn} \chi_{-}(E, F)=\operatorname{sgn}(\mu(F)-\mu(E)) .
$$

ДокАЗАТЕЛЬСТво. При $r(E) r(F) \neq 0$ утверждение непосредственно следует из формулы (6). При $r(E)=0$, т.е. при $E=\mathscr{O}_{\ell}(m), d(E)=-K \cdot \ell=1$, и $(7)$ влечет $\chi_{-}(E, F)=-r(F)$. Аналогично при $r(F)=0$ имеем $\chi_{-}(E, F)=r(E)$. Отсюда легко вытекает требуемое.

1.5. Исключительные наборы. Напомним, что упорядоченньй набор пучков $\left(E_{1}, \ldots, E_{n}\right)$ называется исключительныцм, если все $E_{j}$ - исключительные пучки и при $1 \leqslant j<k \leqslant n$ имеет место $\operatorname{Ext}^{i}\left(E_{k}, E_{j}\right)=0$ для любого $i$. Аналогично определяется исключительньй набор объектов $D^{b}(S)$.

Мы будем называть блоком исключительный набор пучков, в котором $\operatorname{Ext}^{i}\left(E_{j}, E_{k}\right)=0$ для любого $i$ при $j \neq k$. Ясно, что набор, полученный из блока перестановкой его членов, также является блоком. 
ОПРЕДЕЛЕНИЕ. Назовем $m$-блочныцм набором исключительный набор

$$
\left(\mathscr{E}_{1}, \mathscr{E}_{2}, \ldots, \mathscr{E}_{m}\right)=\left(E_{11}, \ldots, E_{1 \alpha_{1}}, E_{21}, \ldots, E_{2 \alpha_{2}}, \ldots, E_{m 1}, \ldots, E_{m \alpha_{m}}\right),
$$

в котором все поднаборы $\mathscr{E}_{i}=\left(E_{i 1}, \ldots, E_{i \alpha_{i}}\right)$ являются блоками. Типом и структурой такого набора назовем соответственно упорядоченный и неупорядоченньй наборы чисел $\left(\alpha_{1}, \alpha_{2}, \ldots, \alpha_{m}\right)$. Число $\alpha_{i}$ будем иногда называть длиной блока $\mathscr{E}_{i}$.

1.6. ПРЕДЛОЖЕНИЕ. Пусть $\mathscr{E}=\left(E_{1}, \ldots, E_{\alpha}\right)$ - блок; тогда справедливы следующие утверждения:

a) $r\left(E_{1}\right)=\cdots=r\left(E_{\alpha}\right)$;

б) $d\left(E_{1}\right)=\cdots=d\left(E_{\alpha}\right)$

(әти инварианты будут обозначаться соответственно через $r(\mathscr{E})$ (ранг бло$\kappa a \mathscr{E})$ ud( $\mathscr{E}))$

в) если $r(\mathscr{E})=0$, то $\mathscr{E}=\left(\mathscr{O}_{\ell_{1}}(D), \ldots, \mathscr{O}_{\ell_{\alpha}}(D)\right)$, где $\ell_{i}-$ попарно непересекающиеся исключительные кривые;

г) дивизоры $c_{i j}=c_{1}\left(E_{i}\right)-c_{1}\left(E_{j}\right), \quad i \neq j$, удовлетворяют соотношениям $c_{i j}^{2}=-2 u c_{i j} \cdot K_{S}=0$.

ДокАЗАТЕЛЬСтво. Непосредственно из определения следует, что $\chi_{-}\left(E_{j}, E_{k}\right)=0 \quad \forall j \neq k$.

Если $r\left(E_{j}\right)=0$, то $E_{j} \cong \mathscr{O}_{\ell}(m)$ и $c_{1}\left(E_{j}\right)=\ell-(-1)$-кривая, откуда $d\left(E_{j}\right)=1$. Тогда в силу формулы $(7) E_{k}$ не может быть локально свободным, следовательно, он имеет те же $r=0$ и $d=1$.

Пусть теперь $E_{j}$ локально свободен; тогда $E_{k}$ тоже локально свободен, и в силу (6)

$$
\frac{d\left(E_{j}\right)}{r\left(E_{j}\right)}=\frac{d\left(E_{k}\right)}{r\left(E_{k}\right)} .
$$

Но ограничение исключительного расслоения $E$ на гладкую эллиптическую кривую из линейного ряда $\left|-K_{S}\right|-$ простое расслоение, степень которого равна $d(E)$ (см., например, [11]), а для простого расслоения на эллиптической кривой известно [1], что его ранг и степень взаимно просты. Отсюда вытекает справедливость а) и б).

Докажем утверждение в). Имеем $\mathscr{E}=\left(\mathscr{O}_{\ell_{1}}\left(m_{1}\right), \ldots, \mathscr{O}_{\ell_{\alpha}}\left(m_{\alpha}\right)\right)$. Формула (5) при $r(E)=r(F)=0$ дает $\chi(E, F)=-c_{1}(E) \cdot c_{1}(F)$. Следовательно, $\ell_{i} \cdot \ell_{j}=0$, $i \neq j$. Наконец, $\mathscr{O}_{\ell_{i}}\left(m_{i}\right) \cong \mathscr{O}_{\ell_{i}}(D)$, где $D=-\sum_{i=1}^{\alpha} m_{i} \ell_{i}$.

Для доказательства г) рассмотрим два случая.

1) $r(\mathscr{E})=0$. В силу в) имеем $c_{i j}=\ell_{i}-\ell_{j}$, где $\ell_{i}^{2}=\ell_{j}^{2}=\ell_{i} \cdot K_{S}=\ell_{j} \cdot K_{S}=-1$ и $\ell_{i} \cdot \ell_{j}=0$. Утверждение проверяется прямым вычислением.

2) $r(\mathscr{E})=r \neq 0$. Из равенства $\chi\left(E_{i}, E_{i}\right)=1$ с помощю (4) получаем

$$
q\left(E_{i}\right)=\frac{1}{2}\left(\frac{1}{r^{2}}+\frac{c_{1}\left(E_{i}\right)^{2}}{r^{2}}-1\right), \quad i=1, \ldots, \alpha .
$$

Из а) и б) следует, что $\mu\left(E_{i}\right)=\mu\left(E_{j}\right)$. Имеем

$$
\begin{aligned}
0 & =\chi\left(E_{i}, E_{j}\right)=r^{2}\left(1+q\left(E_{i}\right)+q\left(E_{j}\right)-\frac{c_{1}\left(E_{i}\right) \cdot c_{1}\left(E_{j}\right)}{r^{2}}\right) \\
& =1+\frac{1}{2}\left(c_{1}\left(E_{i}\right)-c_{1}\left(E_{j}\right)\right)^{2} .
\end{aligned}
$$


Таким образом, $c_{i j}^{2}=-2$. Равенство $c_{i j} \cdot K_{S}=0$ является непосредственным следствием б). Предложение доказано.

СлеДСТВИЕ. Пусть $(\mathscr{E}, \mathscr{F})=\left(E_{1}, \ldots, E_{\alpha}, F_{1}, \ldots, F_{\beta}\right)$ - двублочный исключительный набор. Тогда $\chi\left(E_{j}, F_{k}\right)=\chi_{-}\left(E_{j}, F_{k}\right)$ не зависит от $j \in\{1, \ldots, \alpha\}$ $u k \in\{1, \ldots, \beta\}$.

Эта величина будет обозначаться $\chi(\mathscr{E}, \mathscr{F})$.

1.7. Перестройки (см.[5]). Пусть $(A, B)$ - исключительная пара объектов $D^{b}(S)$. Рассмотрим объект $L_{A}^{D} B$, дополняюший канонический морфизм $\operatorname{RHom}(A, B) \otimes A \stackrel{\text { can }}{\longrightarrow} B$ до выделенного треугольника

$$
L_{A}^{D} B[-1] \longrightarrow \operatorname{RHom}(A, B) \otimes A \stackrel{\text { can }}{\longrightarrow} B \longrightarrow L_{A}^{D} B
$$

Известно [5], что $\left(L_{A}^{D} B, A\right)$ - исключительная пара в $D^{b}(S)$.

ОПРЕДЕЛЕНИЕ. Левой перестройкой назьвается отображение $(A, B) \mapsto$ $\left(L_{A}^{D} B, A\right)$ множества исключительных пар объектов $D^{b}(S)$ в себя.

Левая перестройка пары $(A, B)$ - это пара $\left(L_{A}^{D} B, A\right)$. Объект $L_{A}^{D} B$ мы будем называть результатом перестройки или результатом переноса, или даже просто переносом (влево) объекта $B$ через $A$.

Пусть теперь $(E, F)$ - исключительная пара пучков на $S$. Известно [11, п. 2.11], что из пространств $\operatorname{Ext}^{i}(E, F)$ может быть отличным от нуля либо только Нот, либо только $\operatorname{Ext}^{1}$. По определению исключительной пары $\operatorname{Ext}^{i}(F, E)=0 \quad \forall i$, откуда

$$
\chi(E, F)=\chi_{-}(E, F)
$$

Используя лемму 1.4, мы получаем описание известной из [5] классификации исключительных пар на языке наклонов. Именно, пара $(E, F)$ принадлежит к одному из следующих типов:

$$
\begin{array}{rll}
\text { hom-пара: } & \operatorname{Hom}(E, F) \neq 0, \quad \operatorname{Ext}^{i}(E, F)=0, \quad i=1,2, & \Longleftrightarrow \mu(E)<\mu(F) ; \\
\text { ехt-пара: } & \operatorname{Ext}^{1}(E, F) \neq 0, \operatorname{Ext}^{i}(E, F)=0, \quad i=0,2, & \Longleftrightarrow \mu(E)>\mu(F) ; \\
\text { нуль-пара: } & \operatorname{Ext}^{i}(E, F)=0 \quad \forall i & \Longleftrightarrow \mu(E)=\mu(F) .
\end{array}
$$

Выделенный треугольник (8) для пары $(A, B)=(\delta E, \delta F)$ редуцируется, таким образом, к одной из следующих точных троек:

$$
\begin{aligned}
& 0 \longrightarrow L_{E} F \longrightarrow \operatorname{Hom}(E, F) \otimes E \longrightarrow F \longrightarrow 0 \quad \text { (деление); } \\
& 0 \longrightarrow \operatorname{Hom}(E, F) \otimes E \longrightarrow F \longrightarrow L_{E} F \longrightarrow 0 \quad \text { (отскок); } \\
& 0 \longrightarrow F \longrightarrow L_{E} F \longrightarrow \operatorname{Ext}^{1}(E, F) \otimes E \longrightarrow 0 \quad \text { (расширение). }
\end{aligned}
$$

Пара $\left(L_{\delta E}^{D} \delta F, \delta E\right)$ объектов $D^{b}(S)$ исключительна, следовательно, этим же свойством обладает и пара пучков $\left(L_{E} F, E\right)$. 
ОПРЕДЕЛЕНИЕ. Левой пучковой перестройкой называется отображение $(E, F) \mapsto\left(L_{E} F, E\right)$ множества исключительных пар пучков в себя.

В зависимости от того, какая из вышеприведенных точных троек имеет место, различают три типа пучковых перестроек: деление, отскок и расширение.

Двойственным образом определяются правые перестройки: если $(A, B)$ - исключительная пара в $D^{b}(S)$, то объект $R_{B}^{D} A$ дополняет канонический морфизм $A \rightarrow \operatorname{RHom}^{*}(A, B) \otimes B$ до выделенного треугольника

$$
R_{B}^{D} A \longrightarrow A \longrightarrow \operatorname{RHom}^{*}(A, B) \otimes B \longrightarrow R_{B}^{D} A[1]
$$

и отображение $(A, B) \mapsto\left(B, R_{B}^{D} A\right)$ называется правой перестройкой. При этом пара $\left(B, R_{B}^{D} A\right)$ также исключительна.

Для исключительной пары пучков $(E, F)$ последний выделенный треугольник редуцируется к одной из следующих точных троек:

$$
\begin{array}{lll}
0 \longrightarrow E \longrightarrow \operatorname{Hom}(E, F)^{*} \otimes F \longrightarrow R_{F} E \longrightarrow 0 & \text { (деление); } \\
0 \longrightarrow R_{F} E \longrightarrow E \longrightarrow \operatorname{Hom}(E, F)^{*} \otimes F \longrightarrow 0 & \text { (отскок); } \\
0 \longrightarrow \operatorname{Ext}^{1}(E, F)^{*} \otimes E \longrightarrow R_{F} E \longrightarrow E \longrightarrow 0 & \text { (расширение). }
\end{array}
$$

Правой пучковой перестройкой называется отображение $(E, F) \mapsto\left(F, R_{F} E\right)$ множества исключительных пар в себя.

Имеет место следующее очевидное утверждение о связи перестроек в производной категории с пучковыми перестройками.

1.8. ПРЕДЛОЖЕНИЕ. Если пучковая перестройка пары $(E, F)$ - отскок или расширение, то $L_{\delta E}^{D} \delta F=\delta L_{E} F$ и $R_{\delta F}^{D} \delta E=\delta R_{F} E$. В случае деления $L_{\delta E}^{D} \delta F=\delta L_{E} F[1] u R_{\delta F}^{D} \delta E=\delta R_{F} E[-1]$.

В дальнейшем мы будем отождествлять категорию когерентных пучков на $S$ с ее образом в $D^{b}(S)$ относительно канонического вложения. Символ $\delta$ часто будет опускаться. При этом соглашении последнее предложение означает, что пучковая перестройка, отличная от деления, совпадает с перестройкой в $D^{b}(S)$, а при делении в $D^{b}(S)$ происходит сдвиг на \pm 1 .

1.9. ПРЕДЛОЖЕНИЕ. Тип левой пучковой перестройки допускает следующее описание в терминах наклонов:

$$
\begin{aligned}
(\text { деление }) & \Longleftrightarrow \mu\left(L_{E} F\right)<\mu(E)<\mu(F) ; \\
(\text { отскок }) & \Longleftrightarrow \mu(E) \leqslant \mu(F) \leqslant \mu\left(L_{E} F\right) ; \\
(\text { расширение }) & \Longleftrightarrow \mu(F) \leqslant \mu\left(L_{E} F\right) \leqslant \mu(E) .
\end{aligned}
$$


ДоКАЗАТЕЛЬСТво. При $\mu(E)=\mu(F)$, т.е. $\operatorname{Ext}^{i}(E, F)=0 \forall i$, левая перестройка пары $(E, F)$ может быть рассмотрена как тривиальный отскок или тривиальное расширение и сводится к перестановке ее членов. Леммы 1.2 и 1.4 дают равенства $\mu\left(L_{E} F\right)=\mu(E)=\mu(F)$.

Пусть теперь $\mu(E) \neq \mu(F)$. Если $\mu(E)<\mu(F)$, т.е. $(E, F)$ - пара типа hom, то левая перестройка этой пары - либо деление, либо отскок. При делении из соответствующей точной тройки видно, что пара $\left(L_{E} F, E\right)$ также типа hom, откуда вытекает требуемое неравенство. При отскоке

$$
\chi_{-}\left(F, L_{E} F\right) \stackrel{1.2}{=} \chi_{-}(E \otimes \operatorname{Hom}(E, F), F)=\operatorname{dim} \operatorname{Hom}(E, F) \cdot \chi_{-}(E, F)>0,
$$

и остается применить лемму 1.4 .

Если же $\mu(E)>\mu(F)$, т.е. пара $(E, F)$ типа ext, то левая перестройка этой пары- расширение. Из соответствуюшей точной тройки видно, что пара $\left(L_{E} F, E\right)$ типа hom, a

$$
\chi_{-}\left(F, L_{E} F\right)=\chi_{-}\left(F, \operatorname{Ext}^{1}(E, F) \otimes E\right)=\operatorname{dim} \operatorname{Ext}^{1}(E, F) \cdot \chi_{-}(F, E)>0,
$$

что завершает доказательство.

1.10. Пусть $\tau=\left(E_{1}, \ldots, E_{n}\right)$ - исключительный набор пучков (или объектов $\left.D^{b}(S)\right)$. Известно (см. [2], [5]), что отображения

$$
\begin{aligned}
& \tau \longmapsto\left(E_{1}, \ldots, E_{i-1}, L_{E_{i}}^{(D)} E_{i+1}, E_{i}, E_{i+2}, \ldots, E_{n}\right), \\
& \tau \longmapsto\left(E_{1}, \ldots, E_{i-1}, E_{i+1}, R_{E_{i+1}}^{(D)} E_{i}, E_{i+2}, \ldots, E_{n}\right),
\end{aligned}
$$

$i=1, \ldots, n-1$, приводят к исключительным наборам. Эти отображения называются перестройками исключительных наборов.

1.11. Спирали. Рассмотрим бесконечное в обе стороны продолжение исключительного набора пучков $\left(E_{1}, \ldots, E_{n}\right)$, заданное индуктивными формулами

$$
E_{i+n}=R_{E_{i+n-1}} \ldots R_{E_{i+1}} E_{i}, \quad E_{-i}=L_{E_{1-i}} \ldots L_{E_{n-1-i}} E_{n-i}, \quad i \geqslant 1 .
$$

Так определенная последовательность $\left\{E_{m}\right\}_{m \in \mathbb{Z}}$ называется (пучковой) спиралью периода п, если

$$
E_{i}=E_{n+i} \otimes K_{S} \quad \forall i \in \mathbb{Z} .
$$

Витком спирали называется любой ее поднабор вида $\left(E_{m+1}, \ldots, E_{m+n}\right)$, где $m \in \mathbb{Z}$.

ОПРЕДЕЛЕНИЕ. Исключительный набор $\left(E_{1}, \ldots, E_{n}\right)$ называется полным, если он порождает $D^{b}(S)$.

Известно [2], что исключительный набор на многообразии с обильным антиканоническим классом является полным тогда и только тогда, когда он является витком спирали.

Далее мы будем изучать полные трехблочные исключительные наборы на поверхностях дель Пещо. 


\section{§2. Перестройки блочных наборов}

2.1. Пусть $(\mathscr{E}, \mathscr{F})=\left(E_{1}, \ldots, E_{\alpha}, F_{1}, \ldots, F_{\beta}\right)$ - двублочный набор. Определим перенос пучка $F_{j}$ через блок $\mathscr{E}:$

$$
L_{\mathscr{E}} F_{j}=L_{E_{1}} \ldots L_{E_{\alpha}} F_{j}, \quad j=1, \ldots, \beta .
$$

Согласно $[5, \S 4]$ имеем $\operatorname{Ext}^{i}\left(L_{\mathscr{E}} F_{j}, L_{\mathscr{E}} F_{k}\right)=\operatorname{Ext}^{i}\left(F_{j}, F_{k}\right)=0 \forall i, \forall j \neq k$. Следовательно, набор пучков

$$
L_{\mathscr{E}} \mathscr{F} \triangleq\left(L_{\mathscr{E}} F_{1}, \ldots, L_{\mathscr{E}} F_{\beta}\right)
$$

является блоком; мы его будем иногда называть переносом блока $\mathscr{F}$ через блок $\mathscr{E}$. Рассмотрим пучки $R_{\mathscr{F}} E_{i}=R_{F_{\beta}} \ldots R_{F_{1}} E_{i}$ и набор $R_{\mathscr{F} \mathscr{E}}=\left(R_{\mathscr{F}} E_{1}, \ldots, R_{\mathscr{F}} E_{\alpha}\right)$, который также является блоком.

Легко видеть, что двублочные наборы $\left(L_{\mathscr{E}} \mathscr{F}, \mathscr{E}\right)$ и $\left(\mathscr{F}, R_{\mathscr{F}} \mathscr{E}\right)$ получены из исходного набора $(\mathscr{E}, \mathscr{F})$ конечным числом перестроек в смысле 1.10 и поэтому являются исключительными.

Мы будем также использовать блок $\left(L_{\mathscr{E}}^{D \mathscr{F}}, \mathscr{E}\right)$, получающийся заменой пучковых перестроек в определении $\left(L_{\mathscr{E}} \mathscr{F}, \mathscr{E}\right)$ на перестройки в производной категории

$$
L_{\mathscr{E}}^{D} \mathscr{F} \triangleq\left(L_{\mathscr{E}}^{D} F_{1}, \ldots, L_{\mathscr{E}}^{D} F_{\beta}\right), \quad \text { где } L_{\mathscr{E}}^{D} F_{j}=L_{E_{1}}^{D} \ldots L_{E_{\alpha}}^{D} F_{j} .
$$

ОПРедЕЛЕниЕ. Отображения $(\mathscr{E}, \mathscr{F}) \mapsto\left(L_{\mathscr{E}} \mathscr{F}, \mathscr{E}\right)$ и $(\mathscr{E}, \mathscr{F}) \mapsto\left(\mathscr{F}, R_{\mathscr{F} \mathscr{E}}\right)$ множества двублочных наборов в себя назовем соответственно левой и правой перестройками двублочных наборов.

2.2. ПРЕДЛОЖЕНИЕ. 1) Пучок $L_{\mathscr{E}} F_{j}$ включается в одну из следующих точных троек:

$$
\begin{aligned}
& \left.0 \longrightarrow L_{\mathscr{E}} F_{j} \longrightarrow \bigoplus_{i=1}^{\alpha}\left(\operatorname{Hom}\left(E_{i}, F_{j}\right) \otimes E_{i}\right) \stackrel{\text { can }}{\longrightarrow} F_{j} \longrightarrow 0 \quad \text { (деление }\right) \\
& 0 \longrightarrow \bigoplus_{i=1}^{\alpha}\left(\operatorname{Hom}\left(E_{i}, F_{j}\right) \otimes E_{i}\right) \stackrel{\text { can }}{\longrightarrow} F_{j} \longrightarrow L_{\mathscr{E}} F_{j} \longrightarrow 0 \quad \text { (oтскок) } \\
& 0 \longrightarrow F_{j} \longrightarrow L_{\mathscr{E}} F_{j} \longrightarrow \bigoplus_{i=1}^{\alpha}\left(\operatorname{Ext}^{1}\left(E_{i}, F_{j}\right) \otimes E_{i}\right) \longrightarrow 0 \quad \text { (расширение). }
\end{aligned}
$$

2) Эти три случая описываются в терминах дискретных инвариантов следующим образом:

$$
\begin{aligned}
(\text { деление }) & \Longleftrightarrow \alpha \chi(\mathscr{E}, \mathscr{F}) r(\mathscr{E})>r(\mathscr{F}) \\
(\text { отскок }) & \Longleftrightarrow \chi(\mathscr{E}, \mathscr{F}) \geqslant 0 \text { и } \alpha \chi(\mathscr{E}, \mathscr{F}) r(\mathscr{E}) \leqslant r(\mathscr{F}), \\
(\text { растирение }) & \Longleftrightarrow \chi(\mathscr{E}, \mathscr{F}) \leqslant 0 .
\end{aligned}
$$

В частности, тип точной тройки $n .1)$ не зависит от $j \in\{1, \ldots, \beta\}$. 
ДокАЗАТЕЛЬСТво. Отождествим категорию когерентных пучков на $S$ с ее образом при каноническом вложении в $D^{b}(S)$. Рассмотрим прямую сумму канонических морфизмов

$$
\bigoplus_{i=1}^{\alpha}\left(\operatorname{RHom}\left(E_{i}, F_{j}\right) \otimes E_{i}\right) \longrightarrow F_{j}
$$

которая дополняется до выделенного треугольника

$$
X[-1] \longrightarrow \bigoplus_{i=1}^{\alpha}\left(\operatorname{RHom}\left(E_{i}, F_{j}\right) \otimes E_{i}\right) \longrightarrow F_{j} \longrightarrow X .
$$

Применяя к нему функтор $\operatorname{RHom}\left(E_{i^{\prime}}, \cdot\right)$ и учитывая, что $\operatorname{RHom}\left(E_{i^{\prime}}, E_{i}\right)=0$ при $i \neq i^{\prime}$, имеем длинную точную последовательность

$$
\begin{aligned}
& \cdots \longrightarrow \operatorname{Hom}_{D^{b}(S)}^{k-1}\left(E_{i^{\prime}}, X\right) \longrightarrow \operatorname{Hom}_{D^{b}(S)}^{k}\left(E_{i^{\prime}}, \operatorname{RHom}\left(E_{i^{\prime}}, F_{j}\right) \otimes E_{i^{\prime}}\right) \\
& \stackrel{f_{k}}{\longrightarrow} \operatorname{Hom}_{D^{b}(S)}^{k}\left(E_{i^{\prime}}, F_{j}\right) \longrightarrow \operatorname{Hom}_{D^{b}(S)}^{k}\left(E_{i^{\prime}}, X\right) \longrightarrow \cdots,
\end{aligned}
$$

в которой все $f_{k}$ - канонические отождествления в силу исключительности $E_{i^{\prime}}$. Следовательно, все пространства $\operatorname{Hom}_{D^{b}(S)}^{k}\left(E_{i^{\prime}}, X\right)$ нулевые, т.е.

$$
\operatorname{RHom}\left(E_{i^{\prime}}, X\right)=0 \quad \forall i^{\prime} \in\{1, \ldots, \alpha\} .
$$

Далее, применяя к $(9)$ функтор $\operatorname{RHom}\left(F_{j}, \cdot\right)$ и учитывая, что $\operatorname{RHom}\left(F_{j}, E_{i}\right)=0$ $\forall i \in\{1, \ldots, \alpha\}$, имеем длинную точную последовательность, состоящую из пространств $\operatorname{Hom}_{D^{b}(S)}^{k}\left(F_{j}, \cdot\right)$, из которой заключаем, что

$$
\operatorname{Hom}_{D^{b}(S)}^{0}\left(F_{j}, F_{j}\right) \cong \operatorname{Hom}^{0}\left(F_{j}, X\right) \cong \mathbb{C}, \quad \operatorname{Hom}_{D^{b}(S)}^{k}\left(F_{j}, X\right)=0, \quad k \neq 0 .
$$

Наконец, применяя $\mathrm{RHom}(\cdot, X)$ к $(9)$, получаем, что

$$
\operatorname{Hom}_{D^{b}(S)}^{0}(X, X) \cong \operatorname{Hom}^{0}\left(F_{j}, X\right) \cong \mathbb{C}, \quad \operatorname{Hom}_{D^{b}(S)}^{k}(X, X)=0, \quad k \neq 0,
$$

т.е. $X$ - исключительньй объект в $D^{b}(S)$.

Пусть $T=\operatorname{Tr}\left(E_{1}, \ldots, E_{\alpha}, F_{j}\right)$ - полная триангулированная подкатегория в $D^{b}(S)$, порожденная соответствующим набором объектов; она содержит $T_{\mathscr{E}}=$ $\operatorname{Tr}\left(E_{1}, \ldots, E_{\alpha}\right)$. Выделенный треугольник (9) и равенство (10) означают, что $X$ лежит в пересечении правого ортогонала $T_{\mathscr{E}}^{\perp}($ см. $[2$, п. 3]) и $T$, которое порождается исключительным объектом $L_{\mathscr{E}} F_{j}$. В силу своей исключительности $X$ квазиизоморфен $L_{\mathscr{E}} F_{j}[p]$ - комплексу, имеющему на $p$-м месте $L_{\mathscr{E}} F_{j}$, а на остальных нули.

Таким образом, каждый объект в (9) имеет только один отличный от нуля пучок когомологий, следовательно, этот выделенный треугольник редуцируется к одной из точных троек, приведенных в условии предложения. Это доказывает п. 1).

Утверждения п. 2) ввиду 1.4 очевидны, за исключением, быть может, того, что при $\alpha \chi(\mathscr{E}, \mathscr{F}) r(\mathscr{E})=r(\mathscr{F})$ не может иметь места деление. Покажем это. Действительно, в противном случае $r\left(L_{\mathscr{E}} \mathscr{F}\right)=0$, и, следовательно, $r(\mathscr{E})=0$, так как пучок кручения не может быть подпучком локально свободного пучка. Следовательно, и $r(\mathscr{F})=0$. Но тогда в силу $(7)$ имеем $\chi(\mathscr{E}, \mathscr{F})=0$, т.е. перестройка является тривиальным отскоком и $L_{\mathscr{E}} F_{j}=F_{j}$. Отметим, что в этом случае набор $(\mathscr{E}, \mathscr{F})$ фактически составляет один блок. Предложение доказано.

Двойственными рассуждениями легко доказываются аналогичные факты для правых перестроек. 
2.3. ПРЕДЛОЖЕНИЕ. 1) Пучок $R_{\mathscr{F}} E_{i}$ включается в одну из следующих точных троек:

$$
\begin{aligned}
& 0 \longrightarrow E_{i} \stackrel{\operatorname{can}}{\longrightarrow} \bigoplus_{i=1}^{\beta}\left(\operatorname{Hom}^{*}\left(E_{i}, F_{j}\right) \otimes F_{j}\right) \longrightarrow R_{\mathscr{F}} E_{i} \longrightarrow 0 \quad \text { (деление), } \\
& \left.0 \longrightarrow R_{\mathscr{F}} E_{i} \longrightarrow E_{i} \stackrel{\operatorname{can}}{\longrightarrow} \bigoplus_{i=1}^{\beta}\left(\operatorname{Hom}^{*}\left(E_{i}, F_{j}\right) \otimes F_{j}\right) \longrightarrow 0 \quad \text { (отскок }\right), \\
& 0 \longrightarrow \bigoplus_{i=1}^{\beta}\left(\operatorname{Ext}^{1}\left(E_{i}, F_{j}\right)^{*} \otimes F_{j}\right) \longrightarrow R_{\mathscr{F}} E_{i} \longrightarrow E_{i} \longrightarrow 0 \quad \text { (расиирение). }
\end{aligned}
$$

2) Эти три случая допускают следующее описание в терминах дискретных инвариантов:

$$
\begin{aligned}
(\text { деление }) & \Longleftrightarrow r(\mathscr{E}) \leqslant \beta \chi(\mathscr{E}, \mathscr{F}) r(\mathscr{F}), \\
(\text { отскок }) & \Longleftrightarrow r(\mathscr{E})>\beta \chi(\mathscr{E}, \mathscr{F}) r(\mathscr{F}) \geqslant 0, \\
(\text { расширение }) & \Longleftrightarrow \chi(\mathscr{E}, \mathscr{F}) \leqslant 0 .
\end{aligned}
$$

В частности, тип точной тройки $n .1)$ не зависит от $i \in\{1, \ldots, \alpha\}$.

Отметим, что в простейшем случае, когда каждый из блоков $\mathscr{E}$ и $\mathscr{F}$ состоит из одного пучка, точные тройки предложений 2.2 и 2.3 совпадают с точными тройками п. 1.7.

2.4. Назовем перестройку двублочного набора $(\mathscr{E}, \mathscr{F})$ делением, отскоком или расширениемв зависимости от типа соответствуюших точных троек, приведенных в условиях двух последних предложений.

Ближайшая наша цель - показать, что перестройка двублочного набора, не являющаяся делением, совпадает с перестройкой в $D^{b}(S)$, а при делении происходит сдвиг градуировки на \pm 1 .

Приведем точные формулировки для левых перестроек; случай правых перестроек оставляем читателю.

Для удобства перенумеруем блок $\mathscr{E}$ в обратном порядке: $\mathscr{E}=\left(E_{\alpha}, \ldots, E_{1}\right)$; тогда $L_{\mathscr{E}} F_{j}=L_{E_{\alpha}} \ldots L_{E_{1}} F_{j}$. Рассмотрим последовательность пучков

$$
L^{0} F_{j}=F_{j}, \quad L^{i} F_{j}=L_{E_{i}} \ldots L_{E_{1}} F_{j}, \quad i=1, \ldots, \alpha .
$$

Отметим, что $L^{\alpha} F_{j}=L_{\mathscr{E}} F_{j}$ - перенос $F_{j}$ через блок $\mathscr{E}$, а $L^{i} F_{j}$ - перенос $F_{j}$ через блок $\mathscr{E}_{i}=\left(E_{i}, \ldots, E_{1}\right)$. Обозначим через $L^{i}$ левую (пучковую) перестройку пары $\left(E_{i}, L^{i-1} F_{j}\right)$; тогда пучок $L_{\mathscr{E}} F_{j}$ получается в результате применения последовательности перестроек $L^{1}, \ldots, L^{\alpha}$.

2.5. ПрЕДЛОЖЕНИЕ. 1) Если левая перестройка двублочного набора $(\mathscr{E}, \mathscr{F})$ отлична от деления, то все перестройки $L^{i}, i=1, \ldots, \alpha$, отличны от деления для любого $F_{j} \in \mathscr{F}$.

2) Если левая перестройка набора $(\mathscr{E}, \mathscr{F})$ - деление, то в последовательности перестроек $L^{i}$ делением является ровно одна. 
ДокАЗАТЕЛЬСТво. Пусть $\chi(\mathscr{E}, \mathscr{F})>0$; тогда в силу 2.2 левая перестройка двублочного набора $(\mathscr{E}, \mathscr{F})$ - либо деление, либо отскок. При делении, применяя лемму 1.2, имеем

$$
\begin{gathered}
\chi_{-}\left(L_{\mathscr{E}} F_{j}, \bigoplus_{i=1}^{\alpha} \operatorname{Hom}\left(E_{i}, F_{j}\right) \otimes E_{i}\right)=\chi_{-}\left(\bigoplus_{i=1}^{\alpha} \operatorname{Hom}\left(E_{i}, F_{j}\right) \otimes E_{i}, F_{j}\right) \\
\alpha \chi(\mathscr{E}, \mathscr{F}) \chi\left(L_{\mathscr{E}} \mathscr{F}, \mathscr{E}\right) \\
\alpha \chi(\mathscr{E}, \mathscr{F})^{2}
\end{gathered}
$$

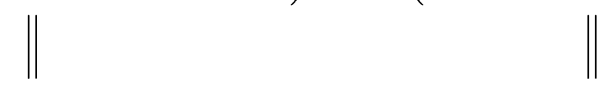

откуда $\chi\left(L_{\mathscr{E}} \mathscr{F}, \mathscr{E}\right)>0$ и $\mu\left(L_{\mathscr{E}} \mathscr{F}\right)<\mu(\mathscr{E})$.

Рассмотрим последовательность $\mu_{i}=\mu\left(L^{i} F_{j}\right)$, где $\mu_{0}=\mu(\mathscr{F})>\mu(\mathscr{E})$ и $\mu_{\alpha}=$ $\mu\left(L_{\mathscr{E}} \mathscr{F}\right)<\mu(\mathscr{E})$. Пусть $p$ - наименьший номер, в котором происходит перемена знака разности $\mu_{i}-\mu(\mathscr{E})$, т.е.

$$
\mu_{p-1}-\mu(\mathscr{E})>0 \quad \text { и } \quad \mu_{p}-\mu(\mathscr{E})<0 .
$$

В силу предложения 1.9 перестройки $L^{i}, 1 \leqslant i \leqslant p-1$, не могут быть ни чем иньм, кроме отскока, а перестройка $L^{p}$ - деление. Но тогда, опять же по предложению 1.9 , все перестройки $L^{i}, p+1 \leqslant i \leqslant \alpha,-$ расширения.

Аналогичным образом, в случае когда левая блочная перестройка набора $(\mathscr{E}, \mathscr{F})$ - отскок, имеем $\mu\left(L_{\mathscr{E}} \mathscr{F}\right)>\mu(\mathscr{F})>\mu(\mathscr{E})$. Из предложения 1.9 вытекает, что в этом случае все $L^{i}$ - отскоки.

Пусть теперь $\chi(\mathscr{E}, \mathscr{F})<0 ; \quad$ тогда вычисления показывают, что $\mu(\mathscr{F})<$ $\mu\left(L_{\mathscr{E}} \mathscr{F}\right)<\mu(\mathscr{E})$, и в силу предложения 1.9 все перестройки $L^{i}$ являются расширениями.

Наконец, при $\chi(\mathscr{E}, \mathscr{F})=0$ все перестройки $L^{i}$ являются, очевидно, тривиальными отскоками (или тривиальными расширениями). Предложение полностью доказано.

СледСТВИЕ. Обгект $X$ в выделенном треугольнике (9) совпадает с $L_{\mathscr{E}}^{D} F_{j}$.

ДоКАЗАТЕЛЬСТВо. Согласно доказательству предложения $2.2 X$ квазиизоморфен $L_{\mathscr{E}}^{D} F_{j}[p]$ для некоторого $p$. Применяя предложения 2.5 и 1.8 , имеем $L_{\mathscr{E}}^{D} F_{j}=$ $L_{\mathscr{E}} F_{j}$, если левая перестройка двублочного набора $(\mathscr{E}, \mathscr{F})$ отлична от деления, и $L_{\mathscr{E}}^{D} F_{j}=L_{\mathscr{E}} F_{j}[1]$, если левая перестройка $(\mathscr{E}, \mathscr{F})$ - деление. Анализируя соответствие между выделенным треугольником (9) и точными тройками из условия предложения 2.2 , получаем требуемое.

2.6. Действие группы кос. Пусть $\tau=\left(\mathscr{E}_{1}, \ldots, \mathscr{E}_{m}\right)-m$-блочный набор. Определим левье и правые перестройки $m$-блочных наборов как отображения множества $m$-блочных наборов в себя:

$$
\begin{aligned}
& L_{i}: \tau \longmapsto\left(\mathscr{E}_{1}, \ldots, \mathscr{E}_{i-1}, L_{\mathscr{E}_{i}} \mathscr{E}_{i+1}, \mathscr{E}_{i}, \mathscr{E}_{i+2}, \ldots, \mathscr{E}_{m}\right), \\
& R_{i}: \tau \longmapsto\left(\mathscr{E}_{1}, \ldots, \mathscr{E}_{i-1}, \mathscr{E}_{i+1}, R_{\mathscr{E}_{i+1}} \mathscr{E}_{i}, \mathscr{E}_{i+2}, \ldots, \mathscr{E}_{m}\right),
\end{aligned}
$$

где $i=1, \ldots, m-1$. Отметим, что структура $m$-блочного набора, определенная в п. 1.5, сохраняется при перестройках $L_{i}$ и $R_{i}$. Следовательно, орбиты действия группы кос содержатся во множествах $m$-блочных наборов данной стуктуры. Перестройки набора $\tau$ в смысле п. 1.10 будем называть не сохраняющими трехблочную структуру. Следуюшее утверждение является аналогом $[2$, п. 2.3$]$ для блочных наборов. 
ПРЕДЛОЖЕНИЕ. 1) Перестройки $R_{i}$ и $L_{i}$ обратны друг другу: $R_{i} \circ L_{i}=\mathrm{id}$.

2) Правые (а также левые) перестройки задают действие группы кос из $m-1$ нитей на множсестве $m$-блочных наборов, т.е. выполняются образующие соотношения группь кос

$$
R_{i} \circ R_{i+1} \circ R_{i}=R_{i+1} \circ R_{i} \circ R_{i+1}, \quad L_{i} \circ L_{i+1} \circ L_{i}=L_{i+1} \circ L_{i} \circ L_{i+1} .
$$

ДокАЗАТЕЛЬСТво. Для доказательства п. 1) достаточно убедиться в том, что для двублочного набора $(\mathscr{E}, \mathscr{F})$ имеет место равенство $R_{\mathscr{E}}\left(L_{\mathscr{E}} \mathscr{F}\right)=\mathscr{F}$. Оно легко следует из того, что левые пучковые перестройки обратны правым.

Для доказательства п. 2) достаточно проверить следующее утверждение: если $(\mathscr{E}, \mathscr{F}, \mathscr{G})$ - трехблочный набор, то

$$
R_{\mathscr{G}}\left(R_{\mathscr{F}} \mathscr{E}\right)=R_{R_{\mathscr{G}} \mathscr{F} \mathscr{E}} .
$$

Но согласно [2] блок, стоящий в левой части последнего равенства, является переносом блока $\mathscr{E}$ вправо через категорию $\operatorname{Tr}(\mathscr{F}, \mathscr{G})=\operatorname{Tr}(\mathscr{G}, R \mathscr{G} \mathscr{F})$ и не зависит от выбора базиса в ней.

2.7. Отметим в заключение этого параграфа, что можно использовать "матричную запись" для перестройки двублочного набора. Например, являюшейся делением левой перестройке набора $(\mathscr{E}, \mathscr{F})$ соответствует последовательность

$$
\begin{aligned}
& \left(\begin{array}{c}
0 \\
\vdots \\
0
\end{array}\right) \rightarrow\left(\begin{array}{c}
L_{\mathscr{E}} F_{1} \\
\vdots \\
L_{\mathscr{E}} F_{\beta}
\end{array}\right) \rightarrow\left[\left(\begin{array}{ccc}
\operatorname{Hom}\left(E_{1}, F_{1}\right) & \ldots & \operatorname{Hom}\left(E_{\alpha}, F_{1}\right) \\
\ldots \ldots \ldots \ldots \ldots \ldots \ldots \ldots \ldots . . . & \ldots \ldots \\
\operatorname{Hom}\left(E_{1}, F_{\beta}\right) & \ldots & \operatorname{Hom}\left(E_{\alpha}, F_{\beta}\right)
\end{array}\right) \odot\left(\begin{array}{c}
E_{1} \\
\vdots \\
E_{\alpha}
\end{array}\right)\right] \\
& \rightarrow\left(\begin{array}{c}
F_{1} \\
\vdots \\
F_{\beta}
\end{array}\right) \rightarrow\left(\begin{array}{c}
0 \\
\vdots \\
0
\end{array}\right)
\end{aligned}
$$

где “произведение" $\odot$ матрицы $\left(\operatorname{Hom}\left(E_{i}, F_{j}\right)\right)$ на столбец пучков есть столбец пучков, имеющий на $j$-м месте

$$
\left(\operatorname{Hom}\left(E_{1}, F_{j}\right) \ldots \operatorname{Hom}\left(E_{\alpha}, F_{j}\right)\right) \odot\left(\begin{array}{c}
E_{1} \\
\vdots \\
E_{\alpha}
\end{array}\right)=\bigoplus_{i=1}^{\alpha}\left(\operatorname{Hom}\left(E_{i}, F_{j}\right) \otimes E_{i}\right) .
$$

Матрищу, состоящую из векторных пространств $\operatorname{Hom}\left(E_{i}, F_{j}\right)$, естественно обозначить $\operatorname{Hom}(\mathscr{E}, \mathscr{F})$; тогда средний член в матричной записи $(12)$ примет вид $\operatorname{Hom}(\mathscr{E}, \mathscr{F}) \odot \mathscr{E}$, и точные последовательности в духе $(12)$, соответствуюшие всевозможным перестройкам набора $(\mathscr{E}, \mathscr{F})$, получатся из последовательностей п. 1.7, описываюших перестройки исключительной пары пучков $(E, F)$, заменой букв $E, F$ на $\mathscr{E}, \mathscr{F}$ и знака тензорного произведения на $\odot$. 


\section{§ 3. Уравнения типа Маркова для полных трехблочных наборов}

Всюду в этом параграфе мы предполагаем, что

$$
(\mathscr{E}, \mathscr{F}, \mathscr{G})=\left(E_{1}, \ldots, E_{\alpha}, F_{1}, \ldots, F_{\beta}, G_{1}, \ldots, G_{\gamma}\right)
$$

- полный трехблочный набор пучков на поверхности дель Пецшо $S$. Полнота эквивалентна тому, что данный упорядоченный набор пучков является витком спирали (cM. 1.11).

3.1. Рассмотрим $\mathbb{Z}$-модуль $\mathrm{K}_{0}(S)$ с билинейной формой $\langle x, y\rangle=\chi(x, y)$; пусть $\lambda: \mathrm{K}_{0}(S) \rightarrow \mathrm{K}_{0}(S)^{*}$ задается формулой $\lambda x=\langle\cdot, x\rangle$. Для любых адлитивных функций $s$ и $t$ на $\mathrm{K}_{0}(S)$ определим

$$
\langle s, t\rangle \triangleq\left\langle\lambda^{-1} s, \lambda^{-1} t\right\rangle
$$

Рассмотрим аддитивные функции $r$ и $d$, где $r-$ ранг, а $d(U)=c_{1}(U) \cdot\left(-K_{S}\right)$. Имеем $\lambda^{-1} r=\mathscr{O}_{p}-$ класс структурного пучка точки. Непосредственным вычислением проверяется, что $\lambda^{-1} d$ лежит в линейной оболочке $\mathscr{O}_{-K_{S}}$ и $\mathscr{O}_{p}$. Таким образом,

$$
\begin{aligned}
& \langle r, r\rangle=\chi\left(\mathscr{O}_{p}, \mathscr{O}_{p}\right)=r\left(\mathscr{O}_{p}\right)=0 \\
& \langle r, d\rangle=\left\langle\mathscr{O}_{p}, \lambda^{-1} d\right\rangle=d\left(\mathscr{O}_{p}\right)=0 \\
& \langle d, r\rangle=r\left(\lambda^{-1} d\right)=0 .
\end{aligned}
$$

Пусть теперь $\left(e_{1}, \ldots, e_{n}\right)$ - полуортогональный базис $\mathrm{K}_{0}(S)$ (т.е. $\left\langle e_{i}, e_{i}\right\rangle=1$, $\left.\left\langle e_{j}, e_{i}\right\rangle=0, j>i\right),\left(e_{n}^{\vee}, \ldots, e_{1}^{\vee}\right)-$ двойственный полуортогональньй базис, т.е. такой, что $\left\langle e_{i}, e_{j}^{\vee}\right\rangle=\delta_{i j}$. Тогда для адлитивных функций $s$ и $t$ имеет место формула

$$
\langle s, t\rangle=\sum_{i=1}^{n} s\left(e_{i}\right) t\left(e_{i}^{\vee}\right) .
$$

Возьмем в качестве $\left(e_{1}, \ldots, e_{n}\right)$ образ набора $(\mathscr{E}, \mathscr{F}, \mathscr{G})$ в $\mathrm{K}_{0}(S)$. Полуортогональность вытекает из исключительности соответствующего набора пучков.

3.2. ПРЕДЛОЖЕНИЕ. Образ набора облектов $D^{b}(S)$

$$
\sigma=\left(G_{\gamma} \otimes K[2], \ldots, G_{1} \otimes K[2], L_{\mathscr{E}}^{D} F_{\beta}, \ldots, L_{\mathscr{E}}^{D} F_{1}, E_{\alpha}, \ldots, E_{1}\right)
$$

в $\mathrm{K}_{0}(S)$ является двойственным базисом $\kappa\left(e_{1}, \ldots, e_{n}\right)$.

ДокАЗАТЕльство. Набор $\sigma$ является исключительным, так как получается из исходного набора $(\mathscr{E}, \mathscr{F}, \mathscr{G})$ перестройками в $D^{b}(S)$. А именно, если ввести единую нумерацию для пучков, входящих в исходньй набор

$$
\left(E_{1}, \ldots, E_{\alpha}, F_{1}, \ldots, F_{\beta}, G_{1}, \ldots, G_{\gamma}\right)=\left(A_{1}, \ldots, A_{n}\right) \subset D^{b}(S),
$$

то из полноты, свойств спирали (п. 1.11) и тривиальности перестроек внутри блока вытекает, что

$$
\sigma=\left(L_{A_{1}} \ldots L_{A_{n-1}} A_{n}, \ldots, L_{A_{1}} L_{A_{2}} A_{3}, L_{A_{1}} A_{2}, A_{1}\right) \triangleq\left(A_{n}^{\vee}, \ldots, A_{1}^{\vee}\right) .
$$


Исключительность $\sigma$ влечет полуортогональность образа $\sigma$ в $\mathrm{K}_{0}(S)$.

Осталось показать, что $\chi\left(A_{i}, A_{j}^{\vee}\right)=\delta_{i j}$.

Рассмотрим наборы объектов $D^{b}(S)$ :

$$
\begin{gathered}
\left(G_{\gamma} \otimes K[2], \ldots, G_{1} \otimes K[2], E_{1}, \ldots, E_{\alpha}, F_{1}, \ldots, F_{\beta}\right), \\
\left(L_{\mathscr{E}}^{D} F_{\beta}, \ldots, L_{\mathscr{E}}^{D} F_{1}, E_{1}, \ldots, E_{\alpha}, G_{1}, \ldots, G_{\gamma}\right) .
\end{gathered}
$$

Легко видеть, что они получены перестройками из исходного набора $(\mathscr{E}, \mathscr{F}, \mathscr{G})$ и поэтому исключительны. Следовательно, имеют место равенства

$$
\begin{aligned}
& \chi\left(E_{i}, L_{\mathscr{E}}^{D} F_{j}\right)=0, \quad \chi\left(F_{i}, E_{j}\right)=0, \quad \chi\left(G_{i}, L_{\mathscr{E}}^{D} F_{j}\right)=0, \\
& \chi\left(E_{i}, G_{j} \otimes K[2]\right)=0, \quad \chi\left(F_{i}, G_{j} \otimes K[2]\right)=0, \quad \chi\left(G_{i}, E_{j}\right)=0, \\
& \chi\left(E_{i}, E_{j}\right)=\delta_{i j} .
\end{aligned}
$$

Последнее легко следует из того, что $\mathscr{E}$ является блоком. В силу следствия п. 2.5 , равенств (11) и ввиду исключительности набора $\left(L_{\mathscr{E}}^{D} F_{j}, E_{1}, \ldots, E_{\alpha}, F_{1}, \ldots\right.$ $\left.\ldots, \widehat{F}_{j}, \ldots, F_{\beta}\right)$ имеем $\chi\left(F_{i}, L_{\mathscr{E}}^{D} F_{j}\right)=\delta_{i j}$. По двойственности Серра $\chi\left(G_{i}, G_{j} \otimes\right.$ $K[2])=\chi\left(G_{j}, G_{i}\right)=\delta_{i j}$, что завершает доказательство.

3.3. Вывод уравнений типа Маркова. Введем обозначения

$$
\begin{gathered}
x=r(\mathscr{E}), \quad y=r(\mathscr{F}), \quad z=r(\mathscr{G}), \quad y^{\prime}=r\left(L_{\mathscr{E}}^{D} \mathscr{F}\right) ; \\
a=\chi(\mathscr{F}, \mathscr{G}), \quad b=\chi(\mathscr{G} \otimes K[2], \mathscr{E})=\chi(\mathscr{G} \otimes K, \mathscr{E}), \quad c=\chi(\mathscr{E}, \mathscr{F}) .
\end{gathered}
$$

Из точных троек предложения 2.2 и следствия п. 2.5 получаем

$$
y^{\prime}=y-c \alpha x, \quad d\left(L_{\mathscr{E}}^{D} \mathscr{F}\right)=d(\mathscr{F})-\operatorname{cod}(\mathscr{E}) .
$$

Будем вычислять значения билинейной формы (13) по формуле (14), используя в качестве базиса в $\mathrm{K}_{0}(S)$ образ исходного набора $(\mathscr{E}, \mathscr{F}, \mathscr{G})$, а в качестве двойственного базиса - образ набора $\sigma$ из предыдущего предложения.

Равенство $\langle r, r\rangle=0$ означает, что $\alpha x^{2}+\beta y y^{\prime}+\gamma z^{2}=0$ или

$$
\alpha x^{2}+\beta y^{2}+\gamma z^{2}=c \alpha \beta x y \text {. }
$$

По предположению $x, y, z \geqslant 0$ и $\alpha, \beta, \gamma>0$. Ранги $x, y, z$ не равны нулю одновременно, так как образ набора $(\mathscr{E}, \mathscr{F}, \mathscr{G})$ в $\mathrm{K}_{0}(S)$ является базисом. Следовательно, $c>0$ и $x, y, z>0$.

Запишем теперь, что дает равенство $\langle r, d\rangle-\langle d, r\rangle=0$ :

$$
\begin{aligned}
\alpha x d(\mathscr{E}) & +\beta y d\left(L_{\mathscr{E}}^{D \mathscr{F}}\right)+\gamma z d(\mathscr{G} \otimes K[2])-\alpha d(\mathscr{E}) x-\beta d(\mathscr{F}) y^{\prime}-\gamma z d(\mathscr{G}) \\
& =\beta(y(d(\mathscr{F})-c \alpha d(\mathscr{E}))-d(\mathscr{F})(y-c \alpha x))+\gamma z(d(\mathscr{G} \otimes K)-d(\mathscr{G})) \\
& =c \alpha \beta(d(\mathscr{F}) x-d(\mathscr{E}) y)-\gamma z^{2} K^{2}=c^{2} \alpha \beta-\gamma z^{2} K^{2}=0 .
\end{aligned}
$$

Отсюда

$$
c=z \sqrt{\frac{K^{2} \gamma}{\alpha \beta}} .
$$

Подставляя это выражение в (15), получаем следуюшее утверждение. 
Teорема. Ранги $x, y, z$ и количества $\alpha, \beta, \gamma$ пучков в блоках полного набора $(\mathscr{E}, \mathscr{F}, \mathscr{G})$ на поверхности дель Пеццо связаны соотношением

$$
\alpha x^{2}+\beta y^{2}+\gamma z^{2}=\sqrt{K^{2} \alpha \beta \gamma} x y z
$$

где $K^{2}$ - квадрат канонического класса поверхности и коэффициент в правой части - целое число.

Поясним последнее утверждение. По доказанному вьше $x, y, z>0$, откуда $\sqrt{K^{2} \alpha \beta \gamma} \in \mathbb{Q}$. Сомножители под корнем целые, следовательно, $\sqrt{K^{2} \alpha \beta \gamma} \in \mathbb{Z}$.

Применяя (16) к пучковым трехблочным виткам $(\mathscr{F}, \mathscr{G}, \mathscr{E} \otimes(-K))$ и $(\mathscr{G} \otimes$ $K, \mathscr{E}, \mathscr{F})$, имеем

$$
a=x \sqrt{\frac{K^{2} \alpha}{\beta \gamma}} \quad \text { и } \quad b=y \sqrt{\frac{K^{2} \beta}{\alpha \gamma}} .
$$

Наконец, выражая $x, y$ и $z$ через $a, b$ и $c$ и подставляя это в (3), получаем уравнение на размерности пространств Нот между блоками:

$$
\frac{a^{2}}{\alpha}+\frac{b^{2}}{\beta}+\frac{c^{2}}{\gamma}=a b c
$$

3.4. СЛЕДСТВИЕ. 1) Для любой пары блоков $(\mathscr{E}, \mathscr{F})$, входящей в полный трехблочный набор, $\operatorname{Hom}\left(E_{i}, F_{j}\right) \neq 0$. Иначе говоря, на поверхностях дель Пецио не существует полных двублочных наборов.

2) Все перестройки полного трехблочного набора - деления.

3) Все пучки, входящие в полный трехблочный набор, локально свободны.

ДокАЗАТЕЛЬСТво. Первое утверждение следует из того, что $c=\chi(\mathscr{E}, \mathscr{F})>0$. Но тогда в паре $(\mathscr{E}, \mathscr{F})$ невозможна перестройка типа расширения, следовательно, невозможна обратная перестройка типа отскока. Последнее утверждение вытекает из классификации исключительных пучков п. 1.3 и полученных выше неравенств $x, y, z>0$.

3.5. Список уравнений. Известно [14], что любая поверхность дель Пецщо $S$ изоморфна либо $\mathbb{P}^{1} \times \mathbb{P}^{1}$, либо $X_{m}$ - плоскости с $m$ раздутыми точками общего положения, где $0 \leqslant m \leqslant 8$.

Пусть на $S$ имеется полный трехблочный набор $(\mathscr{E}, \mathscr{F}, \mathscr{G})$, как и выше; тогда ранги $x, y, z$ положительны и удовлетворяют уравнению (3). Кроме того, образ данного набора в $\mathrm{K}_{0}(S)$ составляет там базис, откуда $\alpha+\beta+\gamma=\operatorname{rk~}_{0}(S)=$ $12-K^{2}$. Таким образом, $\alpha, \beta, \gamma$ и квадрат канонического класса поверхности $S$ удовлетворяют системе условий

$$
\left\{\begin{array}{l}
\alpha+\beta+\gamma+K^{2}=12, \quad K^{2} \alpha \beta \gamma-\text { квадрат целого числа, } \\
\alpha, \beta, \gamma \geqslant 1 \\
1 \leqslant K^{2} \leqslant 9
\end{array}\right.
$$

Система решается конечным перебором. В качестве ответа мы приводим полный список уравнений (3) с указанием поверхностей ${ }^{1}$. Некоторые уравнения можно

\footnotetext{
${ }^{1}$ Ниже мы покажем, что для каждого из этих уравнений существуют соответствующие полные трехблочные наборы.
} 
сократить на общий множитель, но мы этого не делаем, чтобы оставить в качестве коэффициентов именно количества $\alpha, \beta$ и $\gamma$ пучков в соответствуюших блоках. Нетрудно убедиться в том, что с помощью перестроек можно произвольным образом упорядочить блоки с данными количествами пучков. (Например, в первом блоке набора $\left(L_{\mathscr{E}} \mathscr{F}, \mathscr{E}, \mathscr{G}\right)-\beta$ пучков, во втором - $\alpha$, в третьем - $\gamma$ пучков.) Поэтому, не ограничивая общности, мы считаем, что $\alpha \leqslant \beta \leqslant \gamma$.

ОПРЕДЕЛЕНИЕ. Минимальным решением уравнения (3) назовем решение в натуральных числах, имеющее наименьшую сумму $x+y+z$.

ПРЕДЛОЖЕНИЕ. Все уравнения вида (3), имеющие $\alpha \leqslant \beta \leqslant \gamma$, вместе с минимальны.ми решениями собраны в следующей табличе:

\begin{tabular}{|c|c|c|c|}
\hline $\begin{array}{c}\text { Номер } \\
\text { уравнения }\end{array}$ & Поверхность & Уравнение & $\begin{array}{c}\text { Минимальное } \\
\text { решение }\end{array}$ \\
\hline$(\mathbf{1})$ & $\mathbb{P}^{2}$ & $x^{2}+y^{2}+z^{2}=3 x y z$ & $(1,1,1)$ \\
$(\mathbf{2})$ & $\mathbb{P}^{1} \times \mathbb{P}^{1}$ & $x^{2}+y^{2}+2 z^{2}=4 x y z$ & $(1,1,1)$ \\
$(\mathbf{3})$ & $X_{3}$ & $x^{2}+2 y^{2}+3 z^{2}=6 x y z$ & $(1,1,1)$ \\
$(\mathbf{4})$ & $X_{4}$ & $x^{2}+y^{2}+5 z^{2}=5 x y z$ & $(1,2,1)$ и $(2,1,1)$ \\
$(\mathbf{5})$ & $X_{5}$ & $2 x^{2}+2 y^{2}+4 z^{2}=8 x y z$ & $(1,1,1)$ \\
$(\mathbf{6 . 1})$ & $X_{6}$ & $3 x^{2}+3 y^{2}+3 z^{2}=9 x y z$ & $(1,1,1)$ \\
$(\mathbf{6 . 2})$ & $X_{6}$ & $x^{2}+2 y^{2}+6 z^{2}=6 x y z$ & $(2,1,1)$ \\
$(\mathbf{7 . 1})$ & $X_{7}$ & $x^{2}+y^{2}+8 z^{2}=4 x y z$ & $(2,2,1)$ \\
$(\mathbf{7 . 2})$ & $X_{7}$ & $2 x^{2}+4 y^{2}+4 z^{2}=8 x y z$ & $(2,1,1)$ \\
$(\mathbf{7 . 3})$ & $X_{7}$ & $x^{2}+3 y^{2}+6 z^{2}=6 x y z$ & $(3,1,1)$ \\
$(\mathbf{8 . 1})$ & $X_{8}$ & $x^{2}+y^{2}+9 z^{2}=3 x y z$ & $(3,3,1)$ \\
$(\mathbf{8 . 2})$ & $X_{8}$ & $x^{2}+2 y^{2}+8 z^{2}=4 x y z$ & $(4,2,1)$ \\
$(\mathbf{8 . 3})$ & $X_{8}$ & $2 x^{2}+3 y^{2}+6 z^{2}=6 x y z$ & $(3,2,1)$ \\
$(\mathbf{8 . 4})$ & $X_{8}$ & $x^{2}+5 y^{2}+5 z^{2}=5 x y z$ & $(5,2,1)$ и $(5,1,2)$ \\
\hline
\end{tabular}

ДокАЗАТЕЛЬСтво. Мы опускаем проверку того, что в таблице собраны все уравнения вида (3). Покажем, что в правом столбце действительно стоят минимальные решения.

Для уравнений, имеюших решением $(1,1,1)$, утверждение очевидно. Для уравнений $(4),(6.2)$ и (7.2) утверждение верно, поскольку $(1,1,1)$ не является решением этих уравнений. Рассмотрим остальные уравнения.

(7.1): легко видеть, что $x$ и $y$ одинаковой четности, но нечетными они быть не могут, так как $x^{2}+y^{2}$ кратно 4 .

(7.3): $x$ кратно 3 . 
(8.1): $x^{2}+y^{2}$ кратно 3 , но так как вычеты квадратов по модулю 3 принимают значения 0 или 1 , то $x$ и $y$ кратны 3 .

(8.2): $x$ четно, следовательно, $2 y^{2}$ делится на 4 , откуда $y$ четно, и тогда $x^{2}$ кратно 8 , т.е. $x$ кратно 4 .

(8.3): $x$ кратно 3 , а $y$ четно.

(8.4): $x$ кратно 5 ; пусть $x=5 \tilde{x}$, тогда уравнение принимает вид $5 \tilde{x}^{2}+y^{2}+$ $z^{2}=5 \tilde{x} y z$ что совпадает с (4) с точностью до переобозначений.

ЗАмечаниЕ. Поверхность $X_{1}$ имеет $K^{2}=8$, но на ней нет полных трехблочных наборов. Действительно, всего пучков в полном наборе должно быть $\operatorname{dim} \mathrm{K}_{0}\left(X_{1}\right)=4$, т.е. один блок должен был бы состоять из двух пучков. По предложению 1.6 разность $c$ первых классов Чженя этих пучков удовлетворяет соотношениям $c^{2}=-2$ и $c \cdot K=0$. Но таких дивизоров на $X_{1}$ нет.

3.6. Перестройки решений. Пусть $(x, y, z)$ - решение уравнения (3). Перестройкой решений по переменной у назовем отображение

$$
M_{y}:(x, y, z) \longmapsto\left(x, y^{\prime}, z\right),
$$

где

$$
y^{\prime}=\sqrt{\frac{K^{2} \alpha \gamma}{\beta}} x z-y=c \alpha x-y=a \gamma z-y .
$$

Аналогично определяются перестройки решений $M_{x}$ и $M_{z}$ (по $x$ и по $z$ ).

Для любого полного трехблочного набора структуры $\{\alpha, \beta, \gamma\}$ определим соответствующее решение уравнения (3) как тройку чисел $\left(r_{\alpha}, r_{\beta}, r_{\gamma}\right)$, первое из которых - ранг пучков блока длины $\alpha$, и т.д. Если $\alpha<\beta<\gamma$, то одному трехблочному набору соответствует одно решение, а при $\alpha=\beta$ или $\beta=\gamma$ одному трехблочному набору может соответствовать более одного решения. При этом одно и то же решение может соответствовать наборам различных типов, полученных всевозможными перестановками $(\alpha, \beta, \gamma)$.

Пусть $(x, y, z)$ - решение, соответствуюшее набору $(\mathscr{E}, \mathscr{F}, \mathscr{G})$ типа $(\alpha, \beta, \gamma)$; тогда описанное вьше решение $\left(x, y^{\prime}, z\right)$ соответствует наборам $\left(L_{\mathscr{E}} \mathscr{F}, \mathscr{E}, \mathscr{G}\right)$ и $(\mathscr{E}, \mathscr{G}, R \mathscr{G} \mathscr{F})$. Это вытекает из следствия 3.4 и предложения 2.2. Отметим, что $L_{\mathscr{E}} \mathscr{F}=R_{\mathscr{G}} \mathscr{F} \otimes K . \quad$ В общем случае нетрудно проверить, что перестройки набора, изменяюшие блок длины $\alpha$ ( $\beta$ или $\gamma)$, индуцируют перестройки соответствуюшего решения по $x$ ( $y$ или $z)$.

Таким образом, перестройки полных трехблочных наборов согласованы с перестройками соответствуюших решений.

3.7. ПРЕДЛОЖЕНИЕ. а) Любое решение каждого из уравнений из предложения 3.5 приводится перестройками к минимальному решению.

б) Более того, для неминимального решения $(x, y, z)$ одна перестройка уменьшает, а две другие увеличивают сумму $x+y+z$. 
ДоКАЗАТЕЛЬСТво. Для уравнений (1) и (2) утверждение а) хорошо известно (см. [15], [18], [19]). Покажем для этих уравнений справедливость б). В силу симметрии (1) по переменным $x, y$ и $z$ мы можем считать, что $x=\max \{x, y, z\}$. Но тогда $y+y^{\prime}=3 x z \geqslant 3 y z \geqslant 3 y>2 y$, откуда $y^{\prime}>y$, т.е. перестройка по $y$ увеличивает $x+y+z$. Для перестройки по $z$ рассуждения аналогичны.

Уравнение $(\mathbf{2})$ симметрично по $x, y$. Положим для определенности $x \geqslant y$. Рассмотрим случай $x \geqslant z$. Имеем $y+y^{\prime}=4 x z \geqslant 4 y z \geqslant 4 y>2 y \Longrightarrow y^{\prime}>y$, а также $z+z^{\prime}=2 x y \geqslant 2 z y \geqslant 2 z \Longrightarrow z^{\prime} \geqslant z$. Равенство $z^{\prime}=z$ возможно только при $x=z$ и $y=1$. Подставляя эти равенства в $(\mathbf{2})$, имеем $x=1$. Следовательно, если $(x, y, z) \neq(1,1,1)$, то $z^{\prime}>z$. При $x<z$ рассуждения аналогичны.

Рассмотрим теперь уравнения $(\mathbf{3})$ и $(\mathbf{4})$. Введем следующие обозначения:

$$
\Phi(x, y, z)=\alpha x^{2}+\beta y^{2}+\gamma z^{2}-\sqrt{K^{2} \alpha \beta \gamma} x y z, \quad \varphi_{y}(t)=\Phi(x, t, z),
$$

если $(x, y, z)$ - фиксированное решение (3). При этом $t_{1}=y$ и $t_{2}=y^{\prime}$ - корни квадратного уравнения $\varphi_{y}(t)=0$. Мы также будем рассматривать функции $\varphi_{x}(t)=\Phi(t, y, z)$ и $\varphi_{z}(t)=\Phi(x, y, t)$. Основным инструментом доказательства а) будет следующее очевидное утверждение:

$$
y^{\prime} \geqslant y \quad \Longleftrightarrow \quad \varphi_{y}(t) \geqslant 0 \quad \forall t \leqslant y
$$

а также аналогичные утверждения для $x$ и $z$.

Условие $y^{\prime} \geqslant y$ означает, что перестройка решения $(x, y, z)$ по переменной $y$ не уменьшает $y$.

Пусть $(x, y, z)$ - решение $(\mathbf{3})$ такое, что ни одна его перестройка не уменьшает $x+y+z$. Покажем, что тогда $(x, y, z)=(1,1,1)$. Рассмотрим следуюшие случаи:

1) $x \geqslant y \geqslant z$. Имеем $0 \leqslant \varphi_{x}(y)=3 y^{2}+3 z^{2}-6 y^{2} z \leqslant 6 y^{2}-6 y^{2} z$, откуда $z=1$ и $\varphi_{x}(y)=3-3 y^{2} \geqslant 0$. Значит, $y=1$ и из $(\mathbf{3})$ немедленно следует, что $x=1$.

2) $x \geqslant z>y$. Имеем $0 \leqslant \varphi_{x}(z)=4 z^{2}+2 y^{2}-6 z^{2} y<6 z^{2}-6 z^{2} y$, что невозможно.

Остальные случаи также невозможны:

3) $y>x \geqslant z \Longrightarrow 0<\varphi_{y}(x)=3 x^{2}+3 z^{2}-6 x^{2} z \leqslant 6 x^{2}-6 x^{2} z$;

4) $z>x \geqslant y \Longrightarrow 0<\varphi_{z}(x)=4 x^{2}+2 y^{2}-6 x^{2} y \leqslant 6 x^{2}-6 x^{2} y$;

5) $y \geqslant z>x \Longrightarrow 0<\varphi_{y}(z)=x^{2}+5 z^{2}-6 z^{2} x<6 z^{2}-6 z^{2} x$;

6) $z>y>x \Longrightarrow 0<\varphi_{z}(y)=x^{2}+5 y^{2}-6 y^{2} x<6 y^{2}-6 y^{2} x$.

Таким образом, для любого неминимального решения $(\mathbf{3})$ существует перестройка, уменьшающая $x+y+z$. Следовательно, для уравнения (3) утверждение а) доказано.

Доказательство б) проведем при $x=\max \{x, y, z\}$, в остальных случаях рассуждения аналогичны. Имеем $y+y^{\prime}=3 x z \geqslant 3 y z \geqslant 3 y>2 y \Longrightarrow y^{\prime}>y$, а также $z+z^{\prime}=2 x y \geqslant 2 z y \geqslant 2 z \Longrightarrow z^{\prime} \geqslant z$. Равенство $z^{\prime}=z$ возможно только при условиях $x=z$ и $y=1$, из которых следует минимальность решения $(x, y, z)$.

Пусть теперь $(x, y, z)$ - решение (4) такое, что ни одна его перестройка не уменьшает $x+y+z$. Покажем, что $(x, y, z)$ совпадает с одним из минимальных решений $(2,1,1)$ или $(1,2,1)$. Переменные $x$ и $y$ равноправны, поэтому мы полагаем $x \geqslant y$. 
1) $x \geqslant y \geqslant z$. Имеем $0 \leqslant \varphi_{x}(y)=2 y^{2}+5 z^{2}-5 y^{2} z \leqslant 7 y^{2}-5 y^{2} z$, откуда $z=1$ и $\varphi_{x}(y)=5-3 y^{2} \geqslant 0$. Значит, $y=1$, и тогда $x=2$ вытекает непосредственно из (4).

2) $x \geqslant z>y$. Имеем $0 \leqslant \varphi_{x}(z)=6 z^{2}+y^{2}-5 z^{2} y<7 z^{2}-5 z^{2} y$, откуда $y=1$. Рассмотрим перестройку решения по $z: z^{\prime}=x y-z=x-z$. По условию $z^{\prime} \geqslant z$, следовательно, $x \geqslant 2 z$. Тогда $0 \leqslant \varphi_{x}(2 z)=9 z^{2}+1-10 z^{2}=1-z^{2}$, получаем противоречие: $z=1$.

3) $z>x \geqslant y$. Имеем $0 \leqslant \varphi_{z}(x)=6 x^{2}+y^{2}-5 x^{2} y \leqslant 7 x^{2}-5 x^{2} y$, откуда $y=1$ и перестройка решения по $z$ дает $z^{\prime}=x-z<0$. Противоречие.

Следовательно, для любого неминимального решения (4) существует перестройка, уменьшаюшая $x+y+z$, что означает справедливость а). Докажем б), считая по-прежнему $x \geqslant y$.

1) $x \geqslant z$. Имеем $y+y^{\prime}=5 x z \geqslant 5 y z \geqslant 5 y>2 y \Longrightarrow y^{\prime}>y$, т.е. перестройка по $y$ увеличивает $x+y+z$. Пусть $y \geqslant 2$; тогда $z+z^{\prime}=x y \geqslant 2 x \geqslant 2 z$, откуда $z^{\prime} \geqslant z$. Равенство здесь возможно только при условиях $y=2$ и $x=z$, из которых вытекает $x=z=1$. Следовательно, перестройка по $z$ уменьшает $z$ при $(x, y, z) \neq(1,2,1)$. Пусть теперь $y=1$. Покажем, что перестройки по $x$ и по $z$ не могут одновременно уменьшать $x+y+z$. Действительно, если $x^{\prime}=5 z-x<x$, то $5 z<2 x$, откуда $2 z<x$ и $z^{\prime}=x-z<z$.

2) $z>x$. В этом случае $y+y^{\prime}=5 x z \geqslant 5 x y \geqslant 5 y>2 y$, откуда $y^{\prime}>y$, т.е. перестройка по $y$ увеличивает $x+y+z$. Это завершает доказательство б) для (4) в силу симметрии этого уравнения по $x, y$.

Таким образом, предложение справедливо для первых четырех уравнений. Каждое из остальных уравнений сводится к одному из первых четырех заменой переменных, которая в каждом случае возможна (в силу доказательства предложения 3.5$)$ :

$(\mathbf{6 . 2})$ заменой $x=2 \tilde{x}$ приводится к $(\mathbf{3})$;

(7.1) заменой $x=2 \tilde{x}, y=2 \tilde{y}$ приводится к $(\mathbf{2})$;

(7.2) заменой $x=2 \tilde{x}$ приводится к $(2)$;

(7.3) заменой $x=3 \tilde{x}$ приводится к $(3)$;

(8.1) заменой $x=3 \tilde{x}, y=3 \tilde{y}$ приводится к $(\mathbf{1})$;

(8.2) заменой $x=4 \tilde{x}, y=2 \tilde{y}$ приводится к $(\mathbf{2})$;

$(\mathbf{8 . 3})$ заменой $x=3 \tilde{x}, y=2 \tilde{y}$ приводится к $(3)$;

(8.4) заменой $x=5 \tilde{x}$ приводится к (4).

Это завершает доказательство предложения.

3.8. Группы уравнений. Из доказательства предыдущего предложения видно, что все уравнения, начиная с (5), либо получаются из первых четырех уравнений заменой переменных, либо пропорциональны им. Объединим уравнения в следующие группы:

группа I: $(\mathbf{1}),(\mathbf{6 . 1}),(\mathbf{8 . 1})$;

группа II : (2), (5) $,(7.1),(7.2),(8.2)$;

группа III: $(3),(6.2),(\mathbf{7 . 3}),(\mathbf{8 . 3}) ; \quad$ группа IV: $(4),(8.4)$.

Для каждого из уравнений рассмотрим псевдограф, вершинами которого являются решения, и две вершины соединены ребром тогда и только тогда, когда решения 


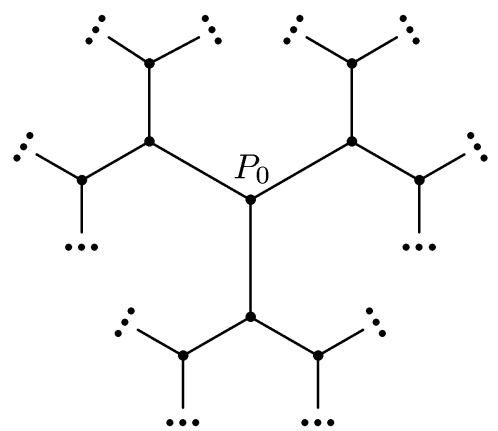

$\Gamma_{1}$

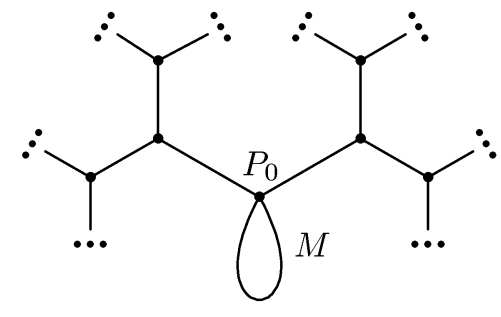

$\Gamma_{2}$

Рис. 1

получены друг из друга одной из перестроек $M_{x}, M_{y}$ или $M_{z}$. Очевидно, что псевдографы решений уравнений, входящих в одну группу, изоморфны. Из предыдущего предложения нетрудно вывести, что псевдографы решений уравнений имеют вид: для группы I - $\Gamma_{1}$ (рис. 1 ), для групп II и III $-\Gamma_{2}$, а для группы IV псевдограф состоит из двух связных компонент, изоморфных $\Gamma_{2}$. Точка $P_{0}$ обозначает минимальное решение. При этом $\Gamma_{1}$ и $\Gamma_{2}$ не содержат циклов, т.е. $\Gamma_{1}$ является графом. Допуская вольность речи, мы будем вместо "псевдограф решений" писать "граф решений" : $\Gamma_{2}$ не является графом из-за наличия единственной петли $M$ (с началом и концом в минимальном решении).

3.9. ОПРЕДЕЛЕНИЕ. Полный трехблочный набор $(\mathscr{E}, \mathscr{F}, \mathscr{G})$ назовем минимальныл $\mathcal{M}$, если сумма $r(\mathscr{E})+r(\mathscr{F})+r(\mathscr{G})$ минимальна среди всех трехблочных наборов такой же структуры.

3.10. ТЕОРЕМА. Любой полный трехблочный набор получается перестройками из минимального набора типа $(\alpha, \beta, \gamma)$, где $\alpha \leqslant \beta \leqslant \gamma$.

ДоКАЗАТЕЛЬСтво. Согласно пп. 3.6 и 3.7 любой полный трехблочньй набор приводится перестройками к минимальному набору. Из обратимости перестроек вытекает, что исходный набор может быть получен из минимального перестройками. Следовательно, достаточно показать, что из минимального набора $(\mathscr{E}, \mathscr{F}, \mathscr{G})$ типа $(\alpha, \beta, \gamma)$, где $\alpha \leqslant \beta \leqslant \gamma$, можно получить минимальный набор, имеющий в качестве типа произвольную перестановку $(\alpha, \beta, \gamma)$. Последовательности перестроек $L_{1} L_{2}$ и $R_{2} R_{1}$ (см. обозначения п. 2.6) приводят $(\mathscr{E}, \mathscr{F}, \mathscr{G})$ к наборам $(\mathscr{G}(K), \mathscr{E}$, $\mathscr{F})$ и $(\mathscr{F}, \mathscr{G}, \mathscr{E}(-K))$ соответственно. Типы этих наборов - циклические перестановки $(\alpha, \beta, \gamma)$. Таким образом, утверждение теоремы справедливо при $\alpha=\beta$ или $\beta=\gamma$. Этому условию удовлетворяют все уравнения, кроме группы III, а для уравнений этой группы перестройка, сохраняющая минимальное решение, индуцируется перестройкой минимального набора, осушествляющей транспозицию двух элементов из $(\alpha, \beta, \gamma)$. Теорема доказана.

Таким образом, вопрос о действии группы кос, в частности о множестве орбит, сводится к вопросу о множестве минимальных наборов. 
В оставшейся части статьи мы исследуем минимальные трехблочные наборы: доказываем их сушествование и описываем действие группы Вейля на наборах данной структуры.

\section{§4. Существование минимальных наборов}

4.1. Соглашения. Зафиксируем на проективной плоскости точки $x_{1}, \ldots, x_{8}$ общего положения и обозначим через $\sigma_{r}: X_{r} \rightarrow \mathbb{P}^{2}$ моноидальное преобразование с центром в $\left\{x_{1}, \ldots, x_{r}\right\}$. Пусть $l$-класс дивизоров на $X_{r}$, являюшийся поднятием класса прямой в $\mathbb{P}^{2}$, и $l_{i}, i=1, \ldots, r,-$ классы исключительных кривых $\sigma_{r}^{-1}\left(x_{i}\right)$. При $1 \leqslant i \leqslant r$ в каждом из $l_{i}$ имеется только один дивизор - сама кривая $\sigma_{r}^{-1}\left(x_{i}\right)$, ее мы будем обозначать тем же символом $l_{i}$.

Поверхность $X_{r+1}$ является раздутием $X_{r}$ в точке $\sigma_{r}^{-1}\left(x_{r+1}\right)$, и мы имеем диаграмму

$$
X_{8} \longrightarrow X_{7} \longrightarrow \cdots \longrightarrow X_{2} \longrightarrow X_{1} \longrightarrow X_{0}=\mathbb{P}^{2} .
$$

$\mathrm{B}$ соответствии с ней будем рассматривать $\mathbb{Z}$-модули $\operatorname{Pic} X_{r}$ вложенными друг в друга:

$$
\mathbb{Z} \cong \operatorname{Pic} \mathbb{P}^{2} \subset \operatorname{Pic} X_{1} \subset \cdots \subset \operatorname{Pic} X_{8}
$$

где $\operatorname{Pic} X_{r}=\mathbb{Z} l \oplus \mathbb{Z} l_{1} \oplus \cdots \oplus \mathbb{Z} l_{r}$. Форма пересечения задается равенствами

$$
l^{2}=1, \quad l_{i}^{2}=-1, \quad i \geqslant 1, \quad l_{i} \cdot l_{j}=0, \quad i \neq j .
$$

Канонический класс поверхности $X_{r}$ имеет вид

$$
\omega_{r}=-3 l+\sum_{i=1}^{r} l_{i}
$$

Обозначим через $\sigma: X_{r} \rightarrow X_{p}$, где $r>p$, композицию морфизмов в диаграмме (18), т.е. раздутие точек $\sigma_{p}^{-1}\left(x_{p+1}\right), \ldots, \sigma_{p}^{-1}\left(x_{r}\right)$. Это не вызовет недоразумений, так как из контекста всегда будет ясно, каковы $p$ и $r$. При наших соглашениях $\operatorname{Pic} X_{p} \subset \operatorname{Pic} X_{r}$ является ортогональным дополнением к линейной оболочке элементов $l_{p+1}, \ldots, l_{r}$.

Каждому классу дивизоров $a l+\sum_{i=1}^{r} b_{i} l_{i}$ по модулю линейной эквивалентности однозначно соответствует класс обратимых пучков по модулю изоморфизма, который мы обозначаем через $\mathscr{O}_{X_{r}}\left(a l+\sum_{i=1}^{r} b_{i} l_{i}\right)$. При этом

$$
\sigma^{*} \mathscr{O}_{X_{p}}\left(a l+\sum_{i=1}^{p} b_{i} l_{i}\right)=\mathscr{O}_{X_{r}}\left(a l+\sum_{i=1}^{p} b_{i} l_{i}\right) .
$$

В этом параграфе мы покажем, что на поверхностях $X_{r}, 3 \leqslant r \leqslant 8$, существуют полные трехблочные исключительные наборы, отвечающие минимальным решениям уравнений типа Маркова (см. п. 3.5), и что все такие наборы получены процедурой, которую можно назвать "поднятием снизу". Общая схема этой процедуры такова. Известно, что если $\tau-$ полный исключительный набор на поверхности $X_{p}$, то набор $\sigma^{*} \tau$, дополненный слева пучками $\mathscr{O}_{l_{p+1}}(-1), \ldots, \mathscr{O}_{l_{r}}(-1)$ (или 
справа пучками $\left.\mathscr{O}_{l_{p+1}}, \ldots, \mathscr{O}_{l_{r}}\right)$, составляет полный исключительный набор на $X_{r}$. При этом, если $\tau$ трехблочный, то последний набор на $X_{r}$ четырехблочный. В некоторых случаях ${ }^{2}$ оказывается возможным сделать такие перестройки четырехблочного набора $\left(\sigma^{*} \tau, \mathscr{O}_{l_{p+1}}, \ldots, \mathscr{O}_{l_{r}}\right)$ (или $\left.\left(\mathscr{O}_{l_{p+1}}(-1), \ldots, \mathscr{O}_{l_{r}}(-1), \sigma^{*} \tau\right)\right)$, чтобы в полученном четырехблочном наборе два соседних блока можно было объединить в один, т.е. фактически получить трехблочный набор на $X_{r}$.

Напомним, что минимальнымм набором на поверхности дель Пещшо мы называем полный трехблочный набор, ранги блоков которого составляют минимальное решение соответствуюшего уравнения типа Маркова.

4.2. ПРЕДЛОЖЕНИЕ. На поверхностях $X_{r}, 3 \leqslant r \leqslant 8$, существуют минимальные наборы типа $(\alpha, \beta, \gamma)$, где $\alpha \leqslant \beta \leqslant \gamma$.

ДокАЗАТЕльство. Можно было бы просто предъявить требуемые наборы, но для дальнейшего важно, что все они получаются вышеописанным "поднятием снизу". Поэтому мы приведем соответствующие последовательности перестроек, пользуясь обозначениями п. 2.6. Если известны дискретные инварианты $r\left(\mathscr{E}_{i}\right)$ и $\chi\left(\mathscr{E}_{i}, \mathscr{E}_{j}\right), i<j$, то предложения 2.2 и 2.3 позволяют установить тип любой перестройки четырехблочного набора $\left(\mathscr{E}_{1}, \mathscr{E}_{2}, \mathscr{E}_{3}, \mathscr{E}_{4}\right)$ и вычислить те же инварианты для перестроенного набора. Отметим, что если набор $\left(\mathscr{E}_{1}, \mathscr{E}_{2}, \mathscr{E}_{3}, \mathscr{E}_{4}\right)$ полньй, то последовательности перестроек

$$
R_{1}^{(3)}=R_{3} \circ R_{2} \circ R_{1} \quad \text { и } \quad L_{3}^{(3)}=L_{1} \circ L_{2} \circ L_{3}
$$

переводят его в наборы $\left(\mathscr{E}_{2}, \mathscr{E}_{3}, \mathscr{E}_{4}, \mathscr{E}_{1}(-K)\right)$ и $\left(\mathscr{E}_{4}(K), \mathscr{E}_{1}, \mathscr{E}_{2}, \mathscr{E}_{3}\right)$ соответственно.

Пункты доказательства будут нумероваться так же, как и уравнения типа Маркова из таблицы п. 3.5. Для экономии места блок, состоящий из пучков кручения $\mathscr{O}_{l_{p}}, \ldots, \mathscr{O}_{l_{r}}, p<r$, будет обозначаться через $\mathscr{L}_{p \div r}$ или через $\mathscr{L}_{p, p+1}$ при $r=p+1$. Итоговый трехблочный набор будет оформляться в виде таблицы

\begin{tabular}{|l|l|l|}
\hline $\mathscr{E}$ & $\mathscr{F}$ & $\mathscr{G}$ \\
\hline
\end{tabular}

причем блок, полученный в результате последнего действия - объединения, будет разделен пунктирной чертой на части, из которых он составлен. Этот блок мы будем называть выделенным. Введем обозначение

$$
\tau_{0}=\left(\mathscr{O}_{\mathbb{P}^{2}}(-1), \mathscr{O}_{\mathbb{P}^{2}}, \mathscr{O}_{\mathbb{P}^{2}}(1)\right)
$$

для хорошо известного витка спирали на $\mathbb{P}^{2}$.

(3). Рассмотрим следующую последовательность перестроек:

$$
\begin{aligned}
& \left(\sigma_{3}^{*} \tau_{0}, \mathscr{L}_{1 \div 3}\right)=\left(\mathscr{O}_{X_{3}}(-l), \mathscr{O}_{X_{3}}, \mathscr{O}_{X_{3}}(l), \mathscr{L}_{1 \div 3}\right) \\
& \stackrel{R_{1}^{(3)}}{\longrightarrow}\left(\mathscr{O}_{X_{3}}, \mathscr{O}_{X_{3}}(l), \mathscr{L}_{1 \div 3}, \mathscr{O}_{X_{3}}\left(2 l-l_{1}-l_{2}-l_{3}\right)\right) \\
& \stackrel{R_{3}}{\longrightarrow} \begin{array}{c|c|c|}
\hline \multirow{O}{*}{\mathscr{O}_{X_{3}}} & \mathscr{O}_{X_{3}}(l) & \mathscr{O}_{X_{3}}\left(2 l-l_{2}-l_{3}\right) \\
& \ldots \ldots \ldots \ldots \ldots \ldots & \mathscr{O}_{X_{3}}\left(2 l-l_{1}-l_{3}\right) \\
& \mathscr{O}_{X_{3}}\left(2 l-l_{1}-l_{2}-l_{3}\right) & \mathscr{O}_{X_{3}}\left(2 l-l_{1}-l_{2}\right) \\
\hline
\end{array}
\end{aligned}
$$

\footnotetext{
${ }^{2}$ А именно, если разность между наклонами каких-либо двух блоков набора $\tau$ равна квадрату канонического класса поверхности $X_{r}$.
} 
Это требуемый набор на плоскости с тремя раздутыми точками.

(4). На поверхности $X_{4}$ имеем

$$
\begin{aligned}
& \left(\sigma_{4}^{*} \tau_{0}, \mathscr{L}_{1 \div 4}\right) \stackrel{R_{1}^{(3)}}{\longrightarrow}\left(\mathscr{O}_{X_{4}}, \mathscr{O}_{X_{4}}(l), \mathscr{L}_{1 \div 4}, \mathscr{O}_{X_{4}}\left(-\omega_{4}-l\right)\right) \\
& \stackrel{R_{3}}{\longrightarrow}\left(\mathscr{O}_{X_{4}}, \mathscr{O}_{X_{4}}(l), \mathscr{O}_{X_{4}}\left(-\omega_{4}-l\right),\left\{\mathscr{O}_{X_{4}}\left(l_{i}-\omega_{4}-l\right)\right\}_{i=1,2,3,4}\right) \\
& \left.\stackrel{L_{2}}{\longrightarrow} \quad \begin{array}{c}
\mathscr{O}_{X_{4}}(l) \\
\mathscr{O}_{X_{4}} \\
\end{array} \quad F \quad \begin{array}{l}
\mathscr{O}_{X_{4}}\left(l_{1}-\omega_{4}-l\right) \\
\mathscr{O}_{X_{4}}\left(l_{2}-\omega_{4}-l\right) \\
\mathscr{O}_{X_{4}}\left(l_{3}-\omega_{4}-l\right) \\
\mathscr{O}_{X_{4}}\left(l_{4}-\omega_{4}-l\right)
\end{array}\right]=\tau_{(4)} .
\end{aligned}
$$

Это набор, отвечающий решению $(1,2,1)$ уравнения $(\mathbf{4})$. Решению $(2,1,1)$ отвечает набор $R_{2} \circ R_{2} \circ R_{1}\left(\tau_{(4)}\right)$.

Расслоение $F$ получается как универсальное расширение

$$
0 \longrightarrow \mathscr{O}_{X_{4}}\left(-\omega_{4}-l\right) \longrightarrow F \longrightarrow \mathscr{O}_{X_{4}}(l) \longrightarrow 0
$$

Имеем $c_{1}(F)=-\omega_{4}, r(F)=2$.

В остальных случаях выпишем только начальный и итоговый наборы и последовательность перестроек. Проверку деталей оставляем заинтересованному читателю.

(5). На поверхности $X_{5}$ четырехблочный набор $\left(\mathscr{L}_{4,5}(-1), \sigma^{*} \tau_{(\mathbf{3})}\right)$ при перестройках $R_{1}^{(3)} \circ R_{1}$ переходит в требуемьй набор

$$
\tau_{(5)}=\begin{array}{|c|c|c|}
\hline & & \mathscr{O}_{X_{5}}\left(-\omega_{5}\right) \\
\mathscr{O}_{X_{5}}\left(l_{4}\right) & \mathscr{O}_{X_{5}}(l) & \ldots \ldots \ldots \ldots \\
\mathscr{O}_{X_{5}}\left(l_{5}\right) & \mathscr{O}_{X_{5}}\left(2 l-l_{1}-l_{2}-l_{3}\right) & \mathscr{O}_{X_{5}}\left(2 l-l_{1}-l_{2}\right) \\
& & \mathscr{O}_{X_{5}}\left(2 l-l_{2}-l_{3}\right) \\
& &
\end{array}
$$

(6.1). Последовательность перестроек $R_{1}^{(3)} \circ L_{3} \circ R_{1}$ переводит четырехблочный набор $\left(\mathscr{L}_{4 \div 6}(-1), \sigma^{*} \tau_{(\mathbf{3})}\right)$ на поверхности $X_{6}$ в искомьй набор

$$
\tau_{(\text {6.1) }}=\begin{array}{|c|c|c|}
\hline \mathscr{O}_{X_{6}}\left(l_{4}\right) & \mathscr{O}_{X_{6}}\left(l-l_{1}\right) & \mathscr{O}_{X_{6}}\left(-\omega_{6}\right) \\
\mathscr{O}_{X_{6}}\left(l_{5}\right) & \mathscr{O}_{X_{6}}\left(l-l_{2}\right) & \mathscr{O}_{X_{6}}(l) \\
\mathscr{O}_{X_{6}}\left(l_{6}\right) & \mathscr{O}_{X_{6}}\left(l-l_{3}\right) & \mathscr{O}_{X_{6}}\left(2 l-l_{1}-l_{2}-l_{3}\right) \\
\hline
\end{array}
$$


(6.2). Четырехблочный набор $\left(\mathscr{L}_{1 \div 6}(-1), \sigma^{*} \tau_{0}\right)$ на $X_{6}$ переводится последовательностью перестроек $R_{1}^{(3)} \circ R_{1}^{(3)} \circ R_{1} \circ R_{2}$ в искомый набор

$$
\tau_{(\text {6.2) }}=\begin{array}{|c|c|cc|}
\hline T_{6} & \mathscr{O}_{X_{6}}(l) & \mathscr{O}_{X_{6}}\left(l_{1}-\omega_{6}\right) & \mathscr{O}_{X_{6}}\left(l_{2}-\omega_{6}\right) \\
& \ldots \ldots \ldots & \mathscr{O}_{X_{6}}\left(l_{3}-\omega_{6}\right) & \mathscr{O}_{X_{6}}\left(l_{4}-\omega_{6}\right) \\
& \mathscr{O}_{X_{6}}\left(-\omega_{6}\right) & \mathscr{O}_{X_{6}}\left(l_{5}-\omega_{6}\right) & \mathscr{O}_{X_{6}}\left(l_{6}-\omega_{6}\right) \\
\hline
\end{array}
$$

Здесь $T_{6}=\sigma_{6}^{*} \mathrm{TP}^{2}(-1), c_{1}\left(T_{6}\right)=l, r\left(T_{6}\right)=2$ и $\mu\left(T_{6}\right)=\frac{3}{2}$.

(7.1). В этом случае найденный авторами путь довольно длинный, и мы разобьем его на две части. Четырехблочный набор $\left(\sigma^{*} \tau_{0}, \mathscr{L}_{1 \div 7}\right)$ переводится последовательностью перестроек $L_{3}^{(3)} \circ L_{3} \circ R_{1}$ в набор

$$
\left(\mathscr{O}_{X_{7}}\left(l+\omega_{7}\right), \mathscr{O}_{X_{7}}, T_{7},\left\{\mathscr{O}_{X_{7}}\left(l-l_{i}\right)\right\}_{i=1, \ldots, 7}\right),
$$

где $T_{7}=\sigma_{7}^{*} \mathrm{TP}^{2}(-1)$ с такими же $r, c_{1}$ и $\mu$, что и $T_{6}$. Последний набор перестройками $R_{1}^{(3)} \circ R_{1}$ приводится к требуемому набору



Расслоение $E_{7}$ получается в результате расширения:

$$
0 \longrightarrow \mathscr{O}_{X_{7}} \longrightarrow E_{7} \longrightarrow \mathscr{O}_{X_{7}}\left(l+\omega_{7}\right) \longrightarrow 0
$$

Имеем $c_{1}\left(E_{7}\right)=l+\omega_{7}, r\left(E_{7}\right)=2$ и $\mu\left(E_{7}\right)=\frac{1}{2}$. В соответствующей последовательности перестроек $R_{1} \circ L_{1}=\mathrm{id}$ :

$$
R_{1}^{(3)} \circ R_{1} \circ\left(L_{1} \circ L_{2} \circ L_{3}\right) \circ L_{3}=R_{1}^{(3)} \circ L_{2} \circ L_{3} \circ L_{3}
$$

(7.2). Последовательность перестроек $L_{3}^{(3)} \circ R_{1} \circ R_{3}$ переводит $\left(\mathscr{L}_{4 \div 7}, \sigma^{*} \tau_{(\mathbf{3})}\right)$ в четырехблочный набор

$$
\left(\begin{array}{cccc} 
& & \mathscr{O}_{X_{7}}\left(l_{4}\right) & \\
\mathscr{O}_{X_{7}}\left(l+\omega_{7}\right) & & \mathscr{O}_{X_{7}}\left(l_{5}\right) & \mathscr{O}_{X_{7}}\left(l-l_{1}\right) \\
\mathscr{O}_{X_{7}}\left(\omega_{7}-l-\omega_{3}\right) & & \mathscr{O}_{X_{7}}\left(l_{6}\right) & \mathscr{O}_{X_{7}}\left(l-l_{2}\right) \\
& & \mathscr{O}_{X_{7}}\left(l_{7}\right) & \mathscr{O}_{X_{7}}\left(l-l_{3}\right)
\end{array}\right)
$$

который при перестройках $R_{1}^{(3)} \circ R_{1}$ переходит в требуемый набор

$$
\tau_{(\mathbf{7 . 2})}=\begin{array}{|c|c|c|}
\hline & \mathscr{O}_{X_{7}}\left(l_{4}\right) & \mathscr{O}_{X_{7}}\left(-\omega_{7}\right) \\
& & \ldots \ldots \ldots \\
E_{7} & \mathscr{O}_{X_{7}}\left(l_{5}\right) & \mathscr{O}_{X_{7}}\left(l-l_{1}\right) \\
E_{7}^{\prime} & \mathscr{O}_{X_{7}}\left(l_{6}\right) & \mathscr{O}_{X_{7}}\left(l-l_{2}\right) \\
& \mathscr{O}_{X_{7}}\left(l_{7}\right) & \mathscr{O}_{X_{7}}\left(l-l_{3}\right) \\
\hline
\end{array}
$$


Здесь $E_{7}$ - расслоение ранга 2 , описанное вьше, а $E_{7}^{\prime}$ - результат правой перестройки пары $\left(\mathscr{O}_{X_{7}}\left(\omega_{7}-l-\omega_{3}\right), \mathscr{O}_{X_{7}}\right)$ :

$$
0 \longrightarrow \mathscr{O}_{X_{7}} \longrightarrow E_{7}^{\prime} \longrightarrow \mathscr{O}_{X_{7}}\left(\omega_{7}-l-\omega_{3}\right) \longrightarrow 0
$$

Имеем $c_{1}\left(E_{7}^{\prime}\right)=\omega_{7}-l-\omega_{3}=-l+l_{4}+l_{5}+l_{6}+l_{7}, r\left(E_{7}^{\prime}\right)=2$ и $\mu\left(E_{7}^{\prime}\right)=\frac{1}{2}$.

(7.3). Четырехблочный набор $\left(\mathscr{O}_{l_{7}}(-1), \sigma^{*} \tau_{(\mathbf{6 . 1})}\right)$ на поверхности $X_{7}$ приводится перестройками $R_{1}^{(3)} \circ R_{1}$ к требуемому набору

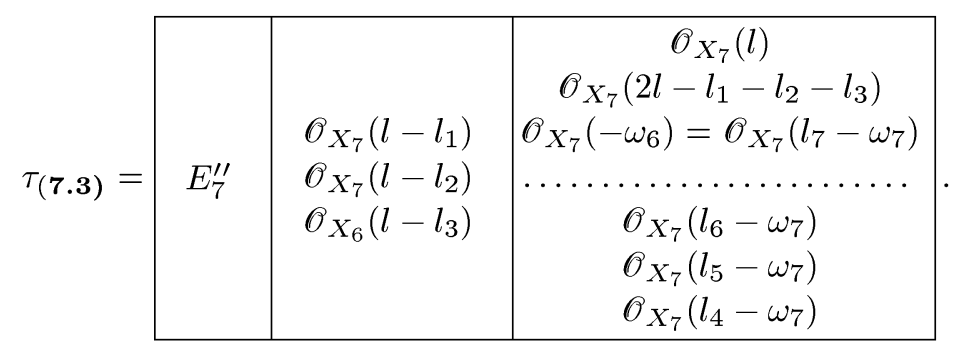

Здесь расслоение $E_{7}^{\prime \prime}$ - результат переноса пучка кручения $\mathscr{O}_{l_{7}}(-1)$ направо через блок $\left(\mathscr{O}_{X_{7}}\left(l_{4}\right), \mathscr{O}_{X_{7}}\left(l_{5}\right), \mathscr{O}_{X_{7}}\left(l_{6}\right)\right)$ :

$$
0 \longrightarrow\left(\mathscr{O}_{X_{7}}\left(l_{4}\right) \oplus \mathscr{O}_{X_{7}}\left(l_{5}\right) \oplus \mathscr{O}_{X_{7}}\left(l_{6}\right)\right) \longrightarrow E_{7}^{\prime \prime} \longrightarrow \mathscr{O}_{l_{7}}(-1) \longrightarrow 0 .
$$

Имеем $c_{1}\left(E_{7}^{\prime \prime}\right)=l_{4}+l_{5}+l_{6}+l_{7}, r\left(E_{7}^{\prime \prime}\right)=3$ и $\mu\left(E_{7}^{\prime \prime}\right)=\frac{4}{3}$.

(8.1). На поверхности $X_{8}$ набор $\left(\sigma^{*}\left(\tau_{0}(-1)\right), \mathscr{L}_{1 \div 8}\right)$ приводится перестройками $L_{3}^{(3)} \circ L_{3} \circ R_{1}$ к четырехблочному набору

$$
\left(\mathscr{O}_{X_{8}}\left(-\omega_{8}\right), \mathscr{O}_{X_{8}}(-l), \sigma^{*}\left(\operatorname{TP}^{2}(-2)\right),\left\{\mathscr{O}_{X_{8}}\left(-l_{i}\right)\right\}_{i=1, \ldots, 8}\right)
$$

Применив к этому последнему набору перестройки $L_{1} \circ L_{1}$, получим требуемый набор

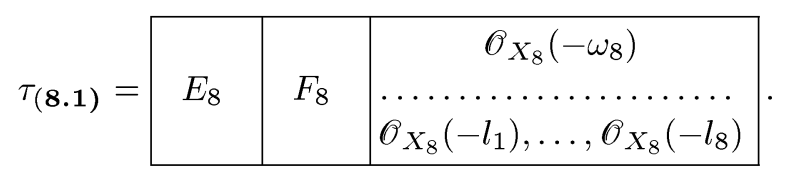

Здесь $E_{8}=L_{\mathscr{O}_{X_{8}}\left(-\omega_{8}\right)} \mathscr{O}_{X_{8}}(-l)$ и $F_{8}=L_{\mathscr{O}_{X_{8}}\left(-\omega_{8}\right)} \sigma^{*}\left(\mathrm{TP}^{2}(-2)\right)$. Обе перестройки - расширения, $c_{1}\left(E_{8}\right)=-2 \omega_{8}-l, c_{1}\left(F_{8}\right)=-\omega_{8}-l, r\left(E_{8}\right)=r\left(F_{8}\right)=3$ и $\mu\left(E_{8}\right)=-\frac{5}{3}, \mu\left(F_{8}\right)=-\frac{4}{3}$.

(8.2). Применив перестройки $L_{3} \circ L_{3}$ к набору $\left(\mathscr{L}_{4 \div 8}(-1), \sigma^{*} \tau_{(\mathbf{3})}\right)$, имеем четырехблочный набор

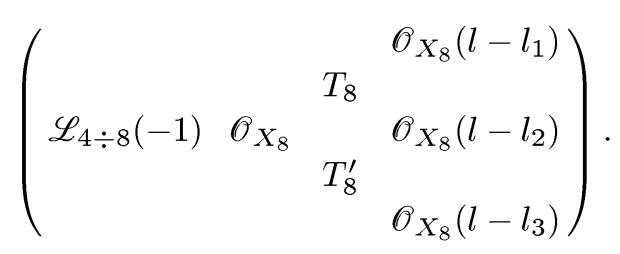


Здесь расслоение $T_{8}=\sigma^{*} \mathrm{TP}^{2}(-1)$ есть левый перенос пучка $\mathscr{O}_{X_{8}}\left(2 l-l_{1}-l_{2}-l_{3}\right)$ через блок $\left\{\mathscr{O}_{X_{8}}\left(l-l_{i}\right)\right\}_{i=1,2,3}$. Это единственное, согласно 1.3 , исключительное расслоение ранга 2 на $X_{8}$, имеющее $c_{1}=l$. Расслоение $T_{8}^{\prime}$ является результатом переноса влево пучка $\mathscr{O}_{X_{8}}(l)$ через тот же блок. Имеем $c_{1}\left(T_{8}^{\prime}\right)=-\omega_{3}-l, r\left(T_{8}^{\prime}\right)=2$ и $\mu\left(T_{8}^{\prime}\right)=\mu\left(T_{8}\right)=\frac{3}{2}$.

Применение к последнему четырехблочному набору перестроек $R_{1}^{(3)} \circ R_{1} \circ R_{1}$ дает искомый набор

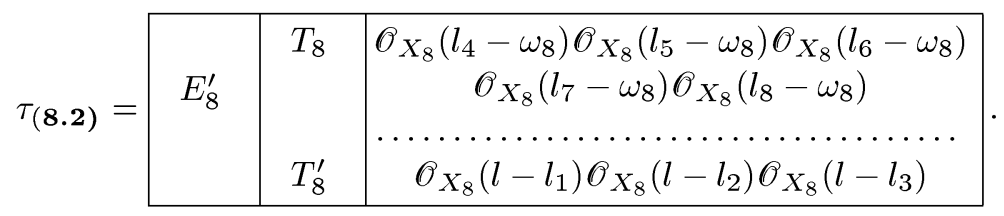

Расслоение $E_{8}^{\prime}$ является переносом направо пучка $\mathscr{O}_{X_{8}}$ через блок $\left\{\mathscr{O}_{X_{8}}\left(l_{i}\right)\right\}_{i=4, \ldots, 8}$. Вычисления показывают, что $c_{1}\left(E_{8}^{\prime}\right)=\sum_{i=4}^{8} l_{i}, r\left(E_{8}^{\prime}\right)=4$ и $\mu\left(E_{8}^{\prime}\right)=1 \frac{1}{4}$.

(8.3). На поверхности $X_{8}$ четырехблочный набор $\left(\mathscr{L}_{7,8}(-1), \sigma^{*} \tau_{(\mathbf{6 . 1})}\right)$ приводится перестройками $R_{1}^{(3)} \circ L_{3} \circ R_{1}$ к требуемому набору

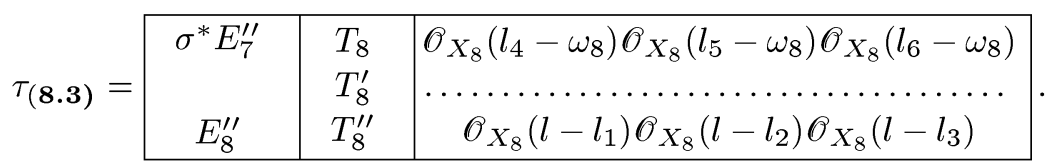

Расслоение $\sigma^{*} E_{7}^{\prime \prime}$ получается в результате переноса направо пучка кручения $\mathscr{O}_{l_{7}}(-1)$ через блок $\left\{\mathscr{O}_{X_{8}}\left(l_{i}\right)\right\}_{i=4,5,6}$. Эта перестройка является поднятием при $\sigma: X_{8} \rightarrow X_{7}$ перестройки, описанной в п.7.3. Расслоение $E_{8}^{\prime \prime}-$ перенос направо пучка $\mathscr{O}_{l_{8}}(-1)$ через тот же блок $\left\{\mathscr{O}_{X_{8}}\left(l_{i}\right)\right\}_{i=4,5,6}$. Соответствующая перестройка является расширением, и мы имеем $c_{1}\left(E_{8}^{\prime \prime}\right)=l_{4}+l_{5}+l_{6}+l_{8}$, $r\left(E_{8}^{\prime}\right)=4$ и $\mu\left(E_{8}^{\prime \prime}\right)=\mu\left(\sigma^{*} E_{7}^{\prime \prime}\right)=\frac{4}{3}$.

Расслоения $T_{8}$ и $T_{8}^{\prime}$ описаны в предыдушем пункте, а $T_{8}^{\prime \prime}$ - перенос влево пучка $\mathscr{O}_{X_{8}}\left(-\omega_{6}\right)$ через блок $\left\{\mathscr{O}_{X_{8}}\left(l-l_{i}\right)\right\}_{i=1,2,3}\left(\right.$ средний блок $\left.\sigma^{*} \tau_{(6.1)}\right)$. Имеем $c_{1}\left(T_{8}^{\prime \prime}\right)=\omega_{6}-\omega_{3}, \quad r\left(T_{8}^{\prime \prime}\right)=2$ и $\mu\left(T_{8}^{\prime \prime}\right)=\mu\left(T_{8}^{\prime}\right)=\mu\left(T_{8}\right)=\frac{3}{2}$.

(8.4). Применив к четырехблочному набору $\left(\sigma^{*} \tau_{(3)}, \mathscr{L}_{4} \div 8\right)$ последовательность перестроек $R_{1}^{(3)} \circ L_{3} \circ R_{1} \circ L_{2}$, получаем набор, отвечаюший решению $(5,2,1)$ :

$$
\tau_{(8.4)}=\begin{array}{|c|c|c|}
\hline & F_{48} & \mathscr{O}_{X_{8}}(l) \\
& F_{58} & \mathscr{O}_{X_{8}}\left(2 l-l_{1}-l_{2}-l_{3}\right) \\
& & \ldots \ldots \ldots \ldots \ldots \ldots \\
& F_{68} & \mathscr{O}_{X_{8}}\left(l-l_{1}-\omega_{8}\right) \\
& F_{78} & \mathscr{O}_{X_{8}}\left(l-l_{2}-\omega_{8}\right) \\
& F_{88} & \mathscr{O}_{X_{8}}\left(l-l_{3}-\omega_{8}\right) \\
\hline
\end{array}
$$

Решению $(5,1,2)$ отвечает набор $R_{2} \circ R_{1} \circ R_{1}\left(\tau_{(8.4)}\right)$. 
Здесь $E_{8}^{\prime \prime \prime}$ - перенос направо $\mathscr{O}_{X_{8}}$ через блок $\left\{\mathscr{O}_{X_{8}}\left(l-l_{i}\right)\right\}_{i=1,2,3}$. Имеем $c_{1}\left(E_{8}^{\prime \prime \prime}\right)=-2 \omega_{3}, r\left(E_{8}^{\prime \prime \prime}\right)=5$ и $\mu\left(E_{8}^{\prime \prime \prime}\right)=2 \frac{2}{5}$. Расслоения $F_{i 8}$ получаются в результате переноса налево пучков кручения $\mathscr{O}_{l_{i}}$ через блок $\left(\mathscr{O}_{X_{8}}(l), \mathscr{O}_{X_{8}}\left(2 l-l_{1}-l_{2}-l_{3}\right)\right)$. Имеем $c_{1}\left(F_{i 8}\right)=-\omega_{3}-l_{i}, i=4,5,6,7,8, r\left(F_{i 8}\right)=2$ и $\mu\left(F_{i 8}\right)=2 \frac{1}{2}$. Это завершает доказательство предложения.

Отметим, что процедура поднятия снизу, которой были получены все минимальные трехблочные наборы в предыдущем доказательстве, обратима. Обратная последовательность действий такова: выделенный блок разбивается на два, и к полученному четырехблочному набору применяется обратная последовательность перестроек. Более того, эти же действия могут быть применены и к произвольному минимальному трехблочному набору $(\mathscr{E}, \mathscr{F}, \mathscr{G})$ на поверхности дель Пецшо, так как совокупность инвариантов $(r(\mathscr{E}), r(\mathscr{F}), r(\mathscr{G})),(\alpha, \beta, \gamma)$ и $(\chi(\mathscr{E}, \mathscr{F}), \chi(\mathscr{F}, \mathscr{G}))$ определяется самим уравнением и, следовательно, принимает те же значения, что и у одного из полученных в предыдушем доказательстве минимальных наборов (см. также (16), (17)). В этом случае ясно, какой блок является выделенным и на какие части (по количеству расслоений) его надо разбивать. Легко видеть, что ранги пучков, входяших в исключительный набор, и размерности пространств Еxt между ними, определяемые с помощъю (6), однозначно вычисляются после перестройки набора. Таким образом, в результате применения вьшеуказанных действий к $(\mathscr{E}, \mathscr{F}, \mathscr{G})$ мы придем к четырехблочному набору, у которого один из блоков состоит из пучков нулевого ранга. Принимая во внимание 1.6, получаем следующее утверждение.

4.3. ПрЕДЛОЖЕНИЕ. Пусть $S$ - поверхность дель Пецио, отличная от $\mathbb{P}^{2}$ $u \mathbb{P}^{1} \times \mathbb{P}^{1}, \tau$ - минимальный трехблочный набор типа $(\alpha, \beta, \gamma), \quad \alpha \leqslant \beta \leqslant \gamma$, на S. Тогда существуют дивизор $D$ на $S$ и последовательность перестроек (не сохраняющих трехблочную структуру), переводящая $\tau(D)$ в набор $\left(\sigma^{*} \tau^{\prime},\left(\mathscr{O}_{e_{1}}, \ldots, \mathscr{O}_{e_{m}}\right)\right)$, әде $e_{1}, \ldots, e_{m}$ - попарно непересекающиеся исключительные кривые, $\sigma: S \rightarrow S^{\prime}-$ морфизм стягивания $\left\{e_{1}, \ldots, e_{m}\right\}$ и $\tau^{\prime}-$ минимальный трехблочный набор на $S^{\prime}$.

\section{§5. Действие группы Вейля на полные трехблочные наборы}

5.1. В этом параграфе через $X_{r}$ обозначается поверхность дель Пецшо степени $9-r$, т.е. мы не фиксируем отождествление $X_{r}$ с плоскостью $\mathrm{c} r$ раздутыми точками. Обозначим через $I_{r} \subset \mathrm{Pic} X_{r}$ множество классов исключительных кривых, которые характеризуются равенствами $e^{2}=e \cdot \omega_{r}=-1$. Пусть $\mathscr{R}_{r} \subset \operatorname{Pic} X_{r}$ множество векторов $s$, имеющих $s^{2}=-2$ и $s \cdot \omega_{r}=0$. Форма пересечения на $\operatorname{Pic} X_{r} \bigotimes_{\mathbb{Z}} \mathbb{R}$, взятая с обратным знаком, индуцирует на ортогональном дополнении к $\omega_{r}$ структуру евклидова пространства. Множество $\mathscr{R}_{r}$ в нем является системой корней. Группа Вейля $W\left(\mathscr{R}_{r}\right)$ этой системы, порожденная симметриями относительно корней, совпадает с группой автоморфизмов решетки $\operatorname{Pic} X_{r}$, сохраняюших $\omega_{r}$ и форму пересечения, а также с группой перестановок элементов из $I_{r}$, сохраняюших их попарные пересечения (см. [14]). Эту группу мы будем обозна- 
чать $W_{r}$ и коротко называть группой Вейля. На $\mathbb{P}^{1} \times \mathbb{P}^{1}$ имеется только один с точностью до знака дивизор с квадратом -2 , т.е. группа Вейля для квадрики есть $\mathbb{Z}_{2}$.

СоГЛАШЕнИЕ. Все формулировки этого параграфа, касающиеся $X_{r} u W_{r}$, кроме предложения 5.4, будут относиться и $\kappa \mathbb{P}^{1} \times \mathbb{P}^{1}$.

Зафиксируем изоморфизм

$$
v: \mathrm{K}_{0}\left(X_{r}\right) \longrightarrow \mathbb{Z} \oplus \operatorname{Pic} X_{r} \oplus \mathbb{Z}, \quad E \stackrel{v}{\longmapsto}\left(r(E), c_{1}(E), 2 \operatorname{ch}_{2}(E)\right),
$$

где $\mathrm{ch}_{2}(E)=c_{1}^{2}(E) / 2-c_{2}(E)-$ вторая компонента характера Черна. Действие групшы Вейля на $\operatorname{Pic} X_{r}$ естественным образом продолжается до действия на $\mathrm{K}_{0}\left(X_{r}\right)$, при этом $r$ и $2 \mathrm{ch}_{2}$ не изменяются. Мы хотим определить действие $W_{r}$ на множестве исключительных наборов, согласованное с действием на $\mathrm{K}_{0}\left(X_{r}\right)$. Положим

$$
g \mathscr{O}_{X_{r}}(D) \triangleq \mathscr{O}_{X_{r}}(g D) \quad \text { и } \quad g \mathscr{O}_{\ell}(m) \triangleq \mathscr{O}_{g \ell}(m) .
$$

5.2. ЛЕмма. Пусть $(E, F) u\left(E^{\prime}, F^{\prime}\right)$ - исключительные пары пучков, причем $v\left(E^{\prime}\right)=g v(E)$ и $v\left(F^{\prime}\right)=g v(F)$. Тогда справедливы следуюшие утверждения:

a) $v\left(R_{F}^{\prime} E^{\prime}\right)=g v\left(R_{F} E\right) u v\left(L_{E}^{\prime} F^{\prime}\right)=g v\left(L_{E} F\right)$;

б) $\operatorname{dim} \operatorname{Ext}^{i}\left(E^{\prime}, F^{\prime}\right)=\operatorname{dim} \operatorname{Ext}^{i}(E, F) \quad \forall i$.

ДокАЗАТЕльство. Пара $(E, F)$ является простейшим двублочным набором. Из предложений 2.2 и 2.3 вытекает, что тип перестроек этой пары зависит только от $r(E), r(F), c_{1}(E) \cdot K$ и $c_{1}(F) \cdot K$ (значение $\chi(E, F)=\chi_{-}(E, F)$ вычисляется по формуле (6)). Действие группы Вейля сохраняет форму пересечения и канонический класс, следовательно, вышеперечисленные инварианты для пары $\left(E^{\prime}, F^{\prime}\right)$ принимают те же значения, что и для $(E, F)$. Таким образом, обе пары имеют одинаковый тип левой и правой перестроек. Утверждение а) проверяется теперь непосредственным вычислением, использующим точные тройки п. 1.7 , аддитивность $v$ и линейность $g$. Справедливость б) вытекает из равенства $\chi(E, F)=\chi\left(E^{\prime}, F^{\prime}\right)$ и классификации исключительных пар (см. п. 1.7). Лемма доказана.

5.3. ПРЕДЛОЖЕНИЕ. Пусть $\tau=\left(E_{1}, \ldots, E_{n}\right)$ - исключительный набор $u g \in W_{r}$. Тогда существует единственный исключительный набор $\tau^{\prime}=$ $\left(E_{1}^{\prime}, \ldots, E_{n}^{\prime}\right)$ такой, что $v\left(E_{i}^{\prime}\right)=g v\left(E_{i}\right)$ для $i=1, \ldots, n$.

Положим $g \tau \triangleq \tau^{\prime}$ u $g E_{i} \triangleq E_{i}^{\prime}$.

ДОКАЗАТЕЛЬСТВО. Согласно $[11,6.11] \tau$ можно включить в полньй исключительный набор, поэтому, не ограничивая общности, мы можем считать $\tau$ полным. По теореме о конструктивности $[11,7.7]$ набор $\tau$ получается некоторой последовательностью перестроек (в смысле 1.10) из полного набора

$$
\tau_{1}=\left(\mathscr{O}_{\ell_{1}}(-1), \ldots, \mathscr{O}_{\ell_{r}}(-1), \mathscr{O}_{X_{r}}, \mathscr{O}_{X_{r}}\left(\ell_{0}\right), \mathscr{O}_{X_{r}}\left(2 \ell_{0}\right)\right),
$$

для которого утверждение очевидным образом выполнено. Применив к полному исключительному набору $g \tau_{1}$, имеем в силу предыдущей леммы требуемый исключительный набор $\tau^{\prime}$. Единственность вытекает из предложения п. 1.3. 
Таким образом, группа Вейля действует на множестве исключительных наборов, сохраняя ранги пучков и размерности взаимных Ext-групп. Это действие согласно 5.2 , а) коммутирует с перестройками. В дальнейшем нас будет интересовать действие $W_{r}$ на полных трехблочных наборах пучков.

5.4. ПРЕДЛОЖЕНИЕ. Пусть $(\mathscr{E}, \mathscr{F}, \mathscr{G})$ u $\left(\mathscr{E}^{\prime}, \mathscr{F}^{\prime}, \mathscr{G}^{\prime}\right)-\partial в а$ минимальных набора типа $(\alpha, \beta, \gamma)$, где $\alpha \leqslant \beta \leqslant \gamma$, отвечающие одному и тому же минимальному решению (әто существенно для уравнений группы IV). Тогда существуют $g \in W_{r}$ и дивизор $D \in \operatorname{Pic} X_{r}$ такие, что

$$
(\mathscr{E}, \mathscr{F}, \mathscr{G})=g\left(\mathscr{E}^{\prime}(D), \mathscr{F}^{\prime}(D), \mathscr{G}^{\prime}(D)\right) .
$$

ДоКАЗАТЕЛЬСТВо будем вести индукцией по $r$. При $r=0$ утверждение тривиально ввиду тривиальности группы $W_{0}$. Пусть утверждение верно для всех $p<r$. Согласно 4.3 сушествуют дивизор $D_{1} \in \operatorname{Pic} X_{r}$ и не сохраняюшая трехблочную структуру последовательность перестроек $\Phi$, переводящая $\left(\mathscr{E}\left(D_{1}\right), \mathscr{F}\left(D_{1}\right), \mathscr{G}\left(D_{1}\right)\right)$ в четырехблочный набор вида $\left(\sigma^{*} \tau, \mathscr{L}_{e}\right)$. Здесь $e=\left\{e_{1}, \ldots, e_{r-p}\right\}-$ множество $(-1)$-кривых с $e_{i} \cdot e_{j}=0$ при $i \neq j$, блок $\mathscr{L}_{e}$ состоит из пучков $\mathscr{O}_{e_{i}}$, $i=1, \ldots, r-p$, морфизм $\sigma: X_{r} \rightarrow X_{p}$ - стягивание множества $e$ и $\tau$ - минимальньй трехблочный набор на $X_{p}$. Та же последовательность перестроек $\Phi$ переводит $\left(\mathscr{E}^{\prime}\left(D_{2}\right), \mathscr{F}^{\prime}\left(D_{2}\right), \mathscr{G}^{\prime}\left(D_{2}\right)\right)$ для некоторого $D_{2} \in \operatorname{Pic} X_{r}$ в аналогичньй набор $\left(\sigma^{* *} \tau^{\prime}, \mathscr{L}_{e^{\prime}}\right)$, где $e^{\prime}=\left\{e_{1}^{\prime}, \ldots, e_{r-p}^{\prime}\right\}$ - другое множество попарно непересекающихся (-1)-кривых, $\sigma^{\prime}: X_{r} \rightarrow X_{p}$-стягивание $e^{\prime}$ и $\tau^{\prime}$ - минимальный набор на $X_{p}$ того же типа, что и $\tau$.

Рассмотрим некоторый элемент $g_{0} \in W_{r}$, переводящий $e_{i}^{\prime}$ в $e_{i}, i=1, \ldots, r-p$. Тогда

$$
g_{0}\left(\sigma^{*} \tau^{\prime}, \mathscr{L}_{e^{\prime}}\right)=\left(\sigma^{*} \tau^{\prime \prime}, \mathscr{L}_{e}\right) .
$$

Здесь $\tau^{\prime \prime}$ - минимальный набор на $X_{p}$ того же типа, что и $\tau$. По предположению индукции найдутся элемент $h \in W_{p}$ и дивизор $D_{0} \in \operatorname{Pic} X_{p}$ такие, что $\tau=h\left(\tau^{\prime \prime}\left(D_{0}\right)\right)$. Отождествив $W_{p}$ с подгруппой в $W_{r}$, оставляюшей неподвижными все $(-1)$-кривые $e_{i}, i=1, \ldots, r-p$, и $\mathrm{Pic} X_{p}$ с ортогональным дополнением к $e$ в $\mathrm{Pic} X_{r}$ (относительно формы пересечения), имеем

$$
\left(\sigma^{*} \tau, \mathscr{L}_{e}\right)=h\left(\sigma^{*} \tau^{\prime \prime}\left(D_{0}\right), \mathscr{L}_{e}\right)=h\left(\sigma^{*} \tau^{\prime \prime}\left(D_{0}\right), \mathscr{L}_{e}\left(D_{0}\right)\right)
$$

(заметим, что $\mathscr{O}_{e_{i}}\left(D_{0}\right)=\mathscr{O}_{e_{i}}$, так как $e_{i} \cdot D_{0}=0$ ).

Объединяя равенства (19) и (20), получаем

$$
\left(\sigma^{*} \tau, \mathscr{L}_{e}\right)=h\left(\left(g_{0} \sigma^{*} \tau^{\prime}\right)\left(D_{0}\right), \mathscr{L}_{e}\left(D_{0}\right)\right)=h g_{0}\left(\sigma^{\prime *} \tau^{\prime}\left(g_{0}^{-1} D_{0}\right), \mathscr{L}_{e^{\prime}}\left(g_{0}^{-1} D_{0}\right)\right) .
$$

Применив к левой и правой частям этого равенства последовательность перестроек $\Phi^{-1}$ и учитьвая перестановочность перестроек с действием $W_{r}$ и подкрутками, имеем

$$
\left(\mathscr{E}\left(D_{1}\right), \mathscr{F}\left(D_{1}\right), \mathscr{G}\left(D_{1}\right)\right)=h g_{0}\left(\mathscr{E}^{\prime}\left(D_{2}+g_{0}^{-1} D_{0}\right), \mathscr{F}^{\prime}\left(D_{2}+g_{0}^{-1} D_{0}\right), \mathscr{G}^{\prime}\left(D_{2}+g_{0}^{-1} D_{0}\right)\right) .
$$

Отсюда уже легко вытекает требуемое утверждение, достаточно положить $g=h g_{0}$ и $D=D_{2}+g_{0}^{-1} D_{0}+g^{-1} D_{1}$. 
5.5. На множестве полных трехблочных наборов на $X_{r}$ действуют группы:

1) группа $\mathrm{Pic} X_{r}$ - посредством тензорного умножения на обратимый пучок;

2) группа $B(3)$ кос из трех нитей - посредством перестроек;

3) группа Вейля $W_{r}$.

При этом действие группы кос перестановочно с действиями Рic $X_{r}$ и $W_{r}$.

Назовем два трехблочых набора эквивалентными, если они получаются друг из друга подкруткой. Предыдушее предложение означает, что $W_{r}$ действует транзитивно на классах эквивалентности минимальных наборов типа $(\alpha, \beta, \gamma)$, где $\alpha \leqslant \beta \leqslant \gamma$. По теореме 3.10 в любой орбите относительно $B(3)$ имеются минимальные наборы указанного типа. Следовательно, $W_{r}$ действует транзитивно на $B(3) \times \operatorname{Pic} X_{r}$-орбитах полных трехблочных наборов данной структуры.

Для краткости орбитой полного трехблочного набора мы будем называть множество всех наборов, полученных из него перестройками и подкрутками, т.е. орбиту относительно действия $B(3) \times \operatorname{Pic} X_{r}$. Орбит полных трехблочных наборов данной структуры конечное число ввиду конечности $W_{r}$. В оставшейся части статьи мы подсчитаем эти числа. Отметим, что структура $\{\alpha, \beta, \gamma\}$ полного трехблочного набора однозначно соответствует уравнению типа Маркова из таблицы п. 3.5.

5.6. ПРЕДЛОЖЕНИЕ. Число $С$ классов әквивалентности минимальных наборов типа $(\alpha, \beta, \gamma)$, где $\alpha \leqslant \beta \leqslant \gamma$, лежащих в одной орбите, конечно. Эти числа приведены в нижней строке следующей таблицы (в верхней-номер уравнения):

\begin{tabular}{|c|c|c|c|c|c|c|}
\hline$(\mathbf{1})$ & $(\mathbf{2})$ & $(\mathbf{3})$ & $(\mathbf{4})$ & $(\mathbf{5})$ & $(\mathbf{6 . 1})$ & $(\mathbf{6 . 2})$ \\
\hline 1 & 1 & 1 & 2 & 2 & 3 & 1 \\
\hline
\end{tabular}

\begin{tabular}{|c|c|c|c|c|c|c|}
\hline$(\mathbf{7 . 1})$ & $(\mathbf{7 . 2})$ & $(\mathbf{7 . 3})$ & $\mathbf{( 8 . 1 )}$ & $(\mathbf{8 . 2})$ & $\mathbf{( 8 . 3 )}$ & $(\mathbf{8 . 4})$ \\
\hline 2 & 2 & 1 & 1 & 1 & 1 & 2 \\
\hline
\end{tabular}

ДокАЗАТЕЛЬСтво. Для уравнения (1) утверждение вытекает из следующего известного факта: любой исключительньй набор на $\mathbb{P}^{2}$, состояший из обратимых пучков, имеет вид $\left(\mathscr{O}_{\mathbb{P}^{2}}(m-1), \mathscr{O}_{\mathbb{P}^{2}}(m), \mathscr{O}_{\mathbb{P}^{2}}(m+1)\right)$. Для уравнения $(\mathbf{2})$ утверждение также хорошо известно, оно вытекает из приведенного в $[5$, п. 5.6$]$ описания исключительных наборов на $\mathbb{P}^{1} \times \mathbb{P}^{1}$, состоящих из обратимых пучков. Рассмотрим остальные уравнения.

Основным инструментом доказательства будут перестройки трехблочных спиралей (см. п. 1.11). Спираль $[\mathscr{E}, \mathscr{F}, \mathscr{G}]$, порожденная полным трехблочным набором $(\mathscr{E}, \mathscr{F}, \mathscr{G})$, представляет собой бесконечную последовательность блоков

$$
(\ldots, \mathscr{E}(K), \mathscr{F}(K), \mathscr{G}(K), \mathscr{E}, \mathscr{F}, \mathscr{G}, \mathscr{E}(-K), \mathscr{F}(-K), \mathscr{G}(-K), \ldots) .
$$

Любые три стояшие подряд блока в этой последовательности образуют полньй трехблочный исключительный набор, называемый витком спирали. Очевидно, 
что спираль однозначно задается любым своим витком. Перестройки витков в смысле $\S 2$ определяют перестройки спиралей. Например, перестройками спиралей являются отображения $[\mathscr{E}, \mathscr{F}, \mathscr{G}] \mapsto\left[L_{\mathscr{E}} \mathscr{F}, \mathscr{E}, \mathscr{G}\right]$ и $[\mathscr{E}, \mathscr{F}, \mathscr{G}] \mapsto[\mathscr{E}, \mathscr{G}, R \mathscr{G} \mathscr{F}]$, которые совпадают: $L_{\mathscr{E}} \mathscr{F}=L_{\mathscr{E}} L_{\mathscr{G}} R_{\mathscr{G}} \mathscr{F}=R_{\mathscr{G}} \mathscr{F}$. Ранги и длины блоков любого витка спирали удовлетворяют одному из уравнений (3), и перестройки спиралей индуцируют перестройки решений этого уравнения, т.е. движение по соответствующему графу решений.

ЛЕмма. Пусть последовательность перестроек $\Phi$ трехблочной спирали $[\mathscr{E}, \mathscr{F}, \mathscr{G}]$ индуиирует замкнутый марирут по графу решений, не содержащий петли с началом и концом в минимальном решении. Тогда $\Phi$ сохраняет трехблочную спираль.

ДокАЗАТЕЛЬство. Пусть $\Psi$ - последовательность перестроек решений, индуцированная $\Phi$, и $w$ - решение, являющееся начальной и конечной точкой маршрута. Граф̆ решений не содержит циклов, следовательно, $\Psi=M \Psi_{1} M$. Здесь $M-$ одна из перестроек $M_{x}, M_{y}, M_{z}$ и $\Psi_{1}$ - последовательность перестроек решений, задающая замкнутый маршрут с началом и концом в $M(w)$. Используя индукцию по числу перестроек в $\Psi$, мы можем считать, что последовательность перестроек спиралей, индуцируюшая $\Psi_{1}$, сохраняет спираль $\widetilde{M}[\mathscr{E}, \mathscr{F}, \mathscr{G}]$, где $\widetilde{M}$ индуцирует $M$. Следовательно, $\Phi[\mathscr{E}, \mathscr{F}, \mathscr{G}]=\widetilde{M}^{2}[\mathscr{E}, \mathscr{F}, \mathscr{G}]$. Квадрат перестройки решений по одной из переменных может индуцироваться только перестройками id $=R_{1} L_{1}=$ $L_{1} R_{1}=R_{2} L_{2}=L_{2} R_{2}$ или $R_{2} R_{1}$, или $L_{1} L_{2}$ подходящего витка спирали. Имеем $R_{2} R_{1}\left(\mathscr{E}^{\prime}, \mathscr{F}^{\prime}, \mathscr{G}^{\prime}\right)=\left(\mathscr{F}^{\prime}, \mathscr{G}^{\prime}, \mathscr{E}^{\prime}(-K)\right)$ и $L_{1} L_{2}\left(\mathscr{E}^{\prime}, \mathscr{F}^{\prime}, \mathscr{G}^{\prime}\right)=\left(\mathscr{G}^{\prime}(K), \mathscr{E}^{\prime}, \mathscr{F}^{\prime}\right)$, т.е. все указанные перестройки витка сохраняют спираль. Лемма доказана.

ЗАмЕчАниЕ. Утверждение леммы верно также в предположении, что замкнутый маршрут по графу решений содержит четное число петель с началом и конщом в минимальном решении.

Это нетрудно проверить, используя лемму.

Вернемся к доказательству предложения. Пусть $(\mathscr{E}, \mathscr{F}, \mathscr{G})$ и $\left(\mathscr{E}^{\prime}, \mathscr{F}^{\prime}, \mathscr{G}^{\prime}\right)$ - минимальные наборы типа $(\alpha, \beta, \gamma), \alpha \leqslant \beta \leqslant \gamma$ (отвечающие одному и тому же минимальному решению для уравнений группы IV), и $\Phi$ - последовательность перестроек, переводящая первьй набор во второй. Последовательность $\Psi$ перестроек решений соответствующего уравнения типа Маркова, индуцированная $\Phi$, задает замкнутый маршрут в графе решений, имеющий в качестве начальной и конечной точки минимальное решение. Возможны два случая.

а) Если $\Psi$ содержит четное число петель, то по доказанному выше наборы $(\mathscr{E}, \mathscr{F}$, $\mathscr{G})$ и $\left(\mathscr{E}^{\prime}, \mathscr{F}^{\prime}, \mathscr{G}^{\prime}\right)$ являются витками одной спирали. Для всех уравнений, кроме (6.1), $\alpha<\beta$ или $\beta<\gamma$, и совпадение типов указанных наборов влечет их эквивалентность: $(\mathscr{E}, \mathscr{F}, \mathscr{G})=\left(\mathscr{E}^{\prime}(m K), \mathscr{F}^{\prime}(m K), \mathscr{G}^{\prime}(m K)\right)$.

б) Если $\Psi$ содержит нечетное число петель, то $\Psi=\Psi_{1} M \Psi_{2}$, где $M-$ петля и $\Psi_{1}, \Psi_{2}$ - маршруты с началом и концом в минимальном решении, содержащие четное число петель. Имеем $\Phi=\Phi_{1} \widetilde{M} \Phi_{2}$, где $\Phi_{1}$ и $\Phi_{2}-$ последовательности перестроек трехблочных наборов, индуцируюшие $\Psi_{1}$ и $\Psi_{2}$, а $\widetilde{M}$ индуцирует $M$. 
По доказанному выше $\Phi_{1}$ и $\Phi_{2}$ сохраняют трехблочную спираль, следовательно,

$$
\left[\mathscr{E}^{\prime}, \mathscr{F}^{\prime}, \mathscr{G}^{\prime}\right]=\Phi[\mathscr{E}, \mathscr{F}, \mathscr{G}]=\widetilde{M}[\mathscr{E}, \mathscr{F}, \mathscr{G}]
$$

Проведем рассуждения отдельно для каждой из групп уравнений.

I. Граф решений не содержит петель, следовательно, возможен только случай а).

Для уравнения (6.1) имеем $\alpha=\beta=\gamma=3$, и с точностью до подкрутки на $m K_{X_{6}}$ мы можем считать, что $\left(\mathscr{E}^{\prime}, \mathscr{F}^{\prime}, \mathscr{G}^{\prime}\right)$ совпадает с одним из наборов $(\mathscr{E}, \mathscr{F}, \mathscr{G})$, $(\mathscr{G}(K), \mathscr{E}, \mathscr{F})$ или $(\mathscr{F}(K), \mathscr{G}(K), \mathscr{E})$. Нетрудно проверить, что эти наборы не эквивалентны при $(\mathscr{E}, \mathscr{F}, \mathscr{G})=\tau_{(\mathbf{6 . 1})}$. Следовательно, в силу 5.4 эти наборы не эквивалентны и в обшем случае.

Для уравнения (8.1) утверждение доказано в а).

II. Рассмотрим уравнение $(\mathbf{5})$. В случае а) наборы $(\mathscr{E}, \mathscr{F}, \mathscr{G})$ и $\left(\mathscr{E}^{\prime}, \mathscr{F}^{\prime}, \mathscr{G}^{\prime}\right)$ эквивалентны, а в случае б) действием группы Вейля и подкруткой второго набора на $m K_{X_{5}}$ мы можем добиться того, что $(\mathscr{E}, \mathscr{F}, \mathscr{G})=\tau_{(\mathbf{5})}$ и $\left(\mathscr{E}^{\prime}, \mathscr{F}^{\prime}, \mathscr{G}^{\prime}\right)=R_{2} R_{2} R_{1} \tau_{(\mathbf{5})}$. Непосредственным вычислением проверяется, что наборы $\tau_{(5)}$ и $R_{2} R_{2} R_{1} \tau_{(5)}$ не эквивалентны. Следовательно, в случае б) наборы $(\mathscr{E}, \mathscr{F}, \mathscr{G})$ и $\left(\mathscr{E}^{\prime}, \mathscr{F}^{\prime}, \mathscr{G}^{\prime}\right)$ также не эквивалентны.

Для уравнений (7.1) и (7.2) рассуждения аналогичны.

В уравнении (8.2) имеем $\alpha<\beta<\gamma$. Типы витков сирали, полученной одной перестройкой из $[\mathscr{E}, \mathscr{F}, \mathscr{G}]$, являются нечетными перестановками $(\alpha, \beta, \gamma)$, следовательно, случай б) невозможен.

III. Для всех уравнений этой группы $\alpha<\beta<\gamma$, и рассуждение аналогично приведенному выше для (8.2).

IV. Для уравнений этой группы спиралям $[\mathscr{E}, \mathscr{F}, \mathscr{G}]$ и $\widetilde{M}[\mathscr{E}, \mathscr{F}, \mathscr{G}]$ соответствуют разные минимальные решения, поэтому случай б) здесь также невозможен. Но классов эквивалентности рассматриваемых минимальных наборов имеется два по числу минимальных решений. Это завершает доказательство предложения.

5.7. Подсчет числа орбит. В этом заключительном пункте статьи мы опишем вычисление количества орбит действия $B(3) \times \operatorname{Pic} X_{r}$ на полных трехблочных наборах данной структуры. На $\mathbb{P}^{2}$ и на $\mathbb{P}^{1} \times \mathbb{P}^{1}$ орбита одна, это показано в [4] и в [19]. Во всех остальных случаях мы сначала найдем число $N$ классов эквивалентности минимальных наборов типа $(\alpha, \beta, \gamma)$, где $\alpha \leqslant \beta \leqslant \gamma$. Согласно 4.3 каждый такой набор $\tau$ на поверхности дель Пецщо $S$ подкруткой и последовательностью перестроек, определяемой по типу набора из доказательства предложения 4.2, может быть приведен к виду $\left(\sigma^{*} \tau^{\prime},\left(\mathscr{O}_{e_{1}}, \ldots, \mathscr{O}_{e_{m}}\right)\right)$, где $e_{1}, \ldots, e_{m}$ - попарно непересекающиеся исключительные кривые, $\sigma: S \rightarrow S^{\prime}$-морфизм стягивания $\left\{e_{1}, \ldots, e_{m}\right\}$ и $\tau^{\prime}$ - минимальный трехблочный набор на $S^{\prime}$. Отметим, что указанная последовательность перестроек не сохраняет трехблочную структуру, и перед ее выполнением нужно найти выделенный блок и разбить его на два блока, длина одного из которых равна как раз $m$. Таким образом, имеет место формула

$$
N \cdot\left(\begin{array}{c}
n \\
m
\end{array}\right)=N^{\prime} \cdot\left(\begin{array}{c}
\text { число множеств } \\
\left\{e_{1}, \ldots, e_{m}\right\}, e_{i} \cdot e_{j}=0
\end{array}\right)
$$


где $n$ - длина выделенного блока и $N^{\prime}$ - число классов эквивалентности минимальных наборов на $S^{\prime}$, имеющих тот же тип, что и $\tau^{\prime}$. Вычислив по этой формуле значения $N$ для всех уравнений типа Маркова и разделив их на соответствующие значения $C$ из предложения 5.6, находим число орбит. Результаты вычислений приведены в следующей таблище:

\begin{tabular}{|c|c|c|c|c|c|}
\hline $\begin{array}{c}\text { Номер } \\
\text { уравнения }\end{array}$ & Поверхность & $(\alpha, \beta, \gamma)$ & $N$ & $C$ & $\begin{array}{c}\text { Число } \\
\text { орбит }\end{array}$ \\
\hline$(\mathbf{1})$ & $\mathbb{P}^{2}$ & $(1,1,1)$ & 1 & 1 & 1 \\
$(\mathbf{2})$ & $\mathbb{P}^{1} \times \mathbb{P}^{1}$ & $(1,1,2)$ & 1 & 1 & 1 \\
$(\mathbf{3})$ & $X_{3}$ & $(1,2,3)$ & 1 & 1 & 1 \\
$(\mathbf{4})$ & $X_{4}$ & $(1,1,5)$ & 2 & 2 & 1 \\
$(\mathbf{5})$ & $X_{5}$ & $(2,2,4)$ & 20 & 2 & 10 \\
$(\mathbf{6 . 1})$ & $X_{6}$ & $(3,3,3)$ & 240 & 3 & 80 \\
$(\mathbf{6 . 2})$ & $X_{6}$ & $(1,2,6)$ & 36 & 1 & 36 \\
$(\mathbf{7 . 1})$ & $X_{7}$ & $(1,1,8)$ & 72 & 2 & 36 \\
$(\mathbf{7 . 2})$ & $X_{7}$ & $(2,4,4)$ & 2520 & 2 & 1260 \\
$(\mathbf{7 . 3})$ & $X_{7}$ & $(1,3,6)$ & 672 & 1 & 672 \\
$(\mathbf{8 . 1})$ & $X_{8}$ & $(1,1,9)$ & 1920 & 1 & 1920 \\
$(\mathbf{8 . 2})$ & $X_{8}$ & $(1,2,8)$ & 8640 & 1 & 8640 \\
$(\mathbf{8 . 3})$ & $X_{8}$ & $(2,3,6)$ & 80640 & 1 & 80640 \\
$(\mathbf{8 . 4})$ & $X_{8}$ & $(1,5,5)$ & 96768 & 2 & 38384 \\
\hline
\end{tabular}

\section{Список литературы}

1. Atiyah M. F. Vector bundles over an elliptic curve // Proc. Lond. Math. Soc. 1957. V. VII. P. 414-452.

2. Бондал А.И. Представления ассоциативных алгебр и когерентные пучки // Изв. АН СССР. Сер. матем. 1988. Т. 53. № 1. С. 25-44.

3. Городенцев А. Л. Перестройки исключительных расслоений на $\mathbb{P}^{n} / /$ Изв. АН СССР. Сер. матем. 1988. Т. 52. № 1. С. 3-15.

4. Gorodentsev A.L., Rudakov A.N. Exceptional vector bundles on the projective spaces // Duke Math. J. 1987. V. 54. № 1. P. 115-130.

5. Городенцев А. Л. Исключительные расслоения на поверхностях с подвижным антиканоническим классом // Изв. АН СССР. Сер. матем. 1988. Т. 52. № 4. С. 3-15.

6. Drezet J.-M., Le Potier J. Fibrés stables et fibrés exceptionnels sur $\mathbb{P}_{2} / /$ Ann. scient. ENS. 1985. V. 18. № 2. P. 193-244.

7. Drezet $J .-M$. Fibrés exceptionnels et suite spectrale de Beilinson généralisée sur $\mathbb{P}_{2}(\mathbb{C})$ // Ann. Math. 1986. V. 275. P. 25-48.

8. Drezet J.-M. Fibrés exceptionnels et variétés de modules de faisceaux semi-stables sur $\mathbb{P}_{2}(\mathbb{C}) / /$ J. Reine und Angew. Math. 1987. V. 380. P. 14-58.

9. Drezet J.-M. Variétés de modules extrémales de faisceaux semi-stables sur $\mathbb{P}_{2}(\mathbb{C}) / /$ Ann. Math. 1991. V. 290. № 4. P. 727-770. 
10. Зюзина С. Ю. Конструктивность исключительных пар векторных расслоений на квадрике // Изв. АН СССР. Сер. матем. 1993. Т. 57. № 1. С. 183-191.

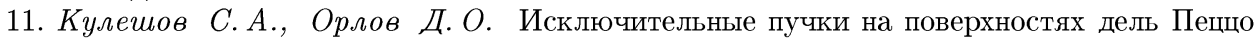
// Изв. РАН. Сер. матем. 1994. Т. 58. №3. С. 53-87.

12. Кулешов C. А. Исключительные и жесткие пучки на поверхностях с антиканоническим классом без базисных компонент / МК НМУ. Препринт № 1. М., 1994.

13. Kuleshov $S$. A. The new proof of the main theorem about exceptional and rigid sheaves on $\mathbb{P}^{2}$. Preprint, MPI /95-11.

14. Манин Ю. И. Кубические формы. М.: Наука, 1972.

15. Марков А. А. О бинарных квадратичных формах положительного определения. СПб., 1880.

16. Ногин Д. Ю. Спирали периода четыре и уравнения типа Маркова // Изв. АН СССР. Сер. матем. 1990. Т. 54. № 4. С. 862-878.

17. Nogin D. Yu. Helices on some Fano threefolds: Constructivity of semiorthogonal bases of $\mathrm{K}_{0} / /$ Ann. ENS. Ser. 4. 1994. V. 27. P. 129-172.

18. Рудаков A. Н. Исключительные расслоения на $\mathbb{P}^{2}$ и числа Маркова // Изв. АН СССР. Сер. матем. 1988. Т. 52. № 1. С. 100-112.

19. Рудаков А. Н. Исключительные расслоения на квадрике // Изв. АН СССР. Сер. матем. 1988. Т. 52. № 4. С. 782-812.

Поступило в редакцию 19.II.1996 